\title{
Compact almost automorphic solutions for semilinear parabolic evolution equations
}

\author{
Brahim Es-sebbar ${ }^{1} \quad$ Khalil Ezzinbi $^{2} \quad$ Kamal Khalii ${ }^{2, *}$
}

April 9, 2020

\author{
${ }^{1}$ Cadi Ayyad University, Faculty of Sciences and Technology Gueliz, Marrakech, Morocco. \\ ${ }^{2}$ Department of Mathematics, Faculty of Sciences Semlalia, Cadi Ayyad University, Marrakesh B.P. \\ 2390-40000, Morocco.
}

\begin{abstract}
In this paper, using the subvariant functional method due to Favard 14, we prove the existence of a unique compact almost automorphic solution for a class of semilinear evolution equations in Banach spaces provided the existence of at least one bounded solution on the right half line. More specifically, we improve the assumptions in [9], we show that the almost automorphy of the coefficients in a weaker sense (Stepanov almost automorphy of order $1 \leq p<\infty$ ) is enough to obtain solutions that are almost automorphic in a strong sense (Bochner almost automorphy). For that purpose we distinguish two cases, $p=1$ and $p>1$. The main difficulty in this work, is to prove the existence of at least one solution with relatively compact range while the forcing term is not necessarily bounded. Moreover, we propose to study a large class of reaction-diffusion problems with unbounded forcing terms.
\end{abstract}

Keywords. Almost automorphic solutions; $C_{0}$-semigroup; Nonlinear evolution equations; Reaction-diffusion equations; Subvariant functional method; Stepanov almost automorphic functions.

\section{Introduction}

The concept of almost automorphy introduced by Bochner 7 is not restricted just to continuous functions. One can generalize that notion to measurable functions with some suitable conditions of integrability, namely, Stepanov almost automorphic functions, see [5, 7, 13. That is a Stepanov almost automorphic function is neither continuous nor bounded necessarily.

Now, consider the following semilinear evolution equation in a Banach space $X$ :

$$
x^{\prime}(t)=A x(t)+f(t, x(t)) \quad \text { for } t \in \mathbb{R},
$$

where $(A, D(A))$ is generator of a $C_{0}$-semigroup $(T(t))_{t \geq 0}$ on $X$. The function $f: \mathbb{R} \times X \longrightarrow X$ is locally integrable of order $1 \leq p<\infty$ in $t$ and continuous in $x$.

The study of existence of almost periodic and almost automorphic solutions to equation (1.1) in infinite dimensional Banach spaces was deeply investigated in the last decades, we refer to 4, 5, 9, 10, 18, 20, 26, 27, 28. Recently, in 4, the authors studied equation (1.1), in the parabolic context, that is when $(A, D(A))$ generates an analytic semigroup $(T(t))_{t>0}$ on a Banach space $X$ which has an exponential dichotomy on $\mathbb{R}$ and $f$ is Stepanov almost periodic of order $1 \leq p<\infty$ and Lipschitzian with respect to $x$. They proved the existence and uniqueness of almost periodic solutions for equation (1.1). In [10, the authors proved the existence and uniqueness of almost automorphic solutions for equation (1.1) in the case where $(A, D(A))$ generates an exponentially stable $C_{0}$-semigroup $(T(t))_{t \geq 0}$ on a Banach space $X$ and $f$ is $S^{p}$-almost automorphic in $t$ (of order $1<p<\infty)$ and Lipschitzian with respect to $x$. Moreover, in $[$, the authors proved the existence of compact almost automorphic solutions for equation (1.1) provided that $(A, D(A))$ generates a compact $C_{0}$-semigroup and $f$ is almost automorphic in the classical sense uniformly with respect to $x$. The results are obtained using the subvariant functional method introduced in [15].

\footnotetext{
*Corresponding author: kamal.khalil@ced.uca.ma
} 
In this paper, using the subvariant functional method, we give sufficient conditions insuring the existence and uniqueness of compact almost automorphic solutions to equation (1.1). We assume that the $C_{0}$-semigroup $(T(t))_{t \geq 0}$ is compact and $f$ is just Stepanov almost automorphic of order $1 \leq p<\infty$. Actually, we begin by introducing a very useful characterization of uniformly Stepanov almost automorphic functions (Lemma 3.1). That is a uniformly $S^{p}$-almost automorphic function is pointwise $S^{p}$-almost automorphic and uniformly continuous on compact sets of $X$ in a weak sense. Then we prove that a mild solution $x$ of equation (1.1) given by:

$$
x(t)=T(t-s) x(s)+\int_{s}^{t} T(t-\sigma) f(\sigma, x(\sigma)) d \sigma \quad \text { for } t \geq s, t, s \in \mathbb{R}
$$

with relatively compact range is uniformly continuous, see Theorem 3.1. Technically, we distinguish two cases, $1<p<\infty$ and $p=1$. Therefore, using Ascoli-Arzela Theorem and an extraction diagonal argument, we construct a mild solution $x$ on $\mathbb{R}$ such that its range is relatively compact, provided that equation (1.1) has at least a mild solution $x_{0}$ on the right half line with relatively compact range satisfying the following inclusion:

$$
\{x(t): t \in \mathbb{R}\} \subset \overline{\left\{x_{0}(t): t \geq t_{0}\right\}},
$$

see Lemma 3.5. Note that, the assumption of existence of mild solutions on the right half line with relatively compact range of equation (1.1) is strongly needed in our work. Consequently, sufficient conditions insuring that assumption are improved in Lemmas 3.6, 3.8 and Proposition 3.1. Here, the situation is quite difficult since $f$ is not necessarily bounded. Indeed, we consider two cases, $1<p<\infty$ and $p=1$. For $1<p<\infty$, in Lemma 3.6, we prove that equation (1.1) has a mild solution on $\mathbb{R}^{+}$with relatively compact range provided it has at least a bounded mild solution on $\mathbb{R}^{+}$. In general, this result does not hold for $p=1$ without any additional assumptions on $f$, see Lemma 3.8 Furthermore, when $f$ satisfies hypothesis (H5) below, the result also holds in general, see Proposition 3.1. After proving that, we give our main results, we show that, if equation (1.1) has at least a bounded mild solution $x_{0}$ on the right half line, then it has a unique compact almost automorphic solution provided the existence of a unique mild solution of equation (1.1) which minimizes a given subvariant functional. Here, we differentiate two cases, $1<p<\infty$, see Theorem 3.2 and the case $p=1$, see Theorem 3.3 .

Furthermore, to illustrate our theoretical results, we study a class of reaction diffusion problems of the form:

$$
\left\{\begin{array}{l}
\frac{\partial}{\partial t} v(t, \xi)=\sum_{k=1}^{n} \frac{\partial^{2}}{\partial \xi_{i}^{2}} v(t, \xi)+g(v(t, \xi))+h(t, \xi) \text { for } t \in \mathbb{R} \text { and } \xi \in \Omega \\
v(t, \xi)=0 \text { for } t \in \mathbb{R}, \xi \in \partial \Omega
\end{array}\right.
$$

in a bounded open domain $\Omega \subset \mathbb{R}^{n}, n \geq 1$ with smooth boundary $\partial \Omega$ and $h: \mathbb{R} \times \Omega \rightarrow \mathbb{R}$ is not necessarily bounded. To satisfy our assumptions, we introduce the main fact that, equation (1.2) has a unique bounded global solution on $\mathbb{R}^{+}$, see Theorem 4.2. Our result is of independent interest and it generalizes many works in the literature, see [17, 23. Therefore, we give our main Theorem of the existence of a unique compact almost automorphic solution to equation (1.2), see Theorem 4.3 .

The work is organized as follows: Section 2 is devoted to preliminaries for Stepanov almost automorphic functions and the subvariant minimizing functional. In Section 3, we give our main results of this work. We begin by the characterization of uniformly Stepanov almost automorphic functions. After that, we prove under the weak assumption of Stepanov almost automorphy on $f$ that every mild solution of (1.1) with relatively compact range is uniformly continuous. In Section 3.1 we give sufficient conditions to the existence of solutions with relatively compact ranges to equation (1.1) on the right half line. Section 3.2 is devoted to main Theorems 3.2 and 3.3 . we prove the existence of compact almost automorphic solutions through a minimizing some subvariant functional provided the existence of a bounded mild solution on the right half line. Finally, Section 4 concerns the application (1.2).

\section{Preliminaries}

Throughout this work, $(X,\|\cdot\|)$ is a Banach space. $B C(\mathbb{R}, X)$ equipped with the supremum norm, the Banach space of bounded continuous functions $f$ from $\mathbb{R}$ into $X$. For $1 \leq p<\infty, q$ denotes 
its conjugate exponent defined by $\frac{1}{p}+\frac{1}{q}=1$ if $p \neq 1$ and $q=\infty$ if $p=1$. By $L_{l o c}^{p}(\mathbb{R}, X)$ (resp. $L^{p}(\mathbb{R}, X)$ ), we designate the space (resp. the Banach space) of all equivalence classes of measurable functions $f$ from $\mathbb{R}$ into $X$ such that $\|f(\cdot)\|^{p}$ is locally integrable (resp. integrable).

\subsection{Almost automorphic functions}

Definition 2.1 (H. Bohr) [8 A continuous function $f: \mathbb{R} \longrightarrow X$ is said to be almost periodic if for every $\varepsilon>0$, there exists $l_{\varepsilon}>0$, such that for every $a \in \mathbb{R}$, there exists $\tau \in\left[a, a+l_{\varepsilon}\right]$ satisfying:

$$
\|f(t+\tau)-f(t)\|<\varepsilon \text { for all } t \in \mathbb{R} .
$$

The space of all such functions is denoted by $A P(\mathbb{R}, X)$.

Definition 2.2 (S. Bochner) 7] A continuous function $f: \mathbb{R} \longrightarrow X$ is called almost automorphic if for every sequence $\left(\sigma_{n}\right)_{n \geq 0}$ of real numbers, there exist a subsequence $\left(s_{n}\right)_{n \geq 0} \subset\left(\sigma_{n}\right)_{n \geq 0}$ and $g: \mathbb{R} \longrightarrow X$ such that, for each $t \in \mathbb{R}$

$$
g(t)=: \lim _{n} f\left(t+s_{n}\right) \quad \text { and } \quad f(t)=\lim _{n} g\left(t-s_{n}\right) .
$$

If the above limits hold uniformly on compact subsets of $\mathbb{R}$, then $f$ is called compact almost automorphic.

In the sequel, $A A(\mathbb{R}, X)$ (resp. $K A A(\mathbb{R}, X)$ ) denotes the space of almost automorphic (resp. compact almost automorphic) $X$-valued functions.

Proposition 2.1 [12] Let $f \in A A(\mathbb{R}, X)$. Then, $f$ is compact almost automorphic if and only if it is uniformly continuous.

Remark 2.1 (i) By the pointwise convergence, the function $g$ in Definition 2.2 is only measurable and bounded but not necessarily continuous. If one of the two convergences in Definition 2.2 is uniform on $\mathbb{R}$, then $f$ is almost periodic. For more details about this topic we refer the reader to the book 21].

(ii) An almost automorphic function may not be uniformly continuous. In fact, the real function

$$
f(t)=\sin \left(\frac{1}{2+\cos (t)+\cos (\sqrt{2} t)}\right) \text { for } t \in \mathbb{R}
$$

belong to $A A(\mathbb{R}, \mathbb{R})$, but is not uniformly continuous.

Then, we have the following inclusions:

$$
A P(\mathbb{R}, X) \subset K A A(\mathbb{R}, X) \subset A A(\mathbb{R}, X) \subset B C(\mathbb{R}, X) .
$$

Definition 2.3 [13] Let $1 \leq p<\infty$. A function $f \in L_{l o c}^{p}(\mathbb{R}, X)$ is said to be bounded in the sense of Stepanov if

$$
\sup _{t \in \mathbb{R}}\left(\int_{[t, t+1]}\|f(s)\|^{p} d s\right)^{\frac{1}{p}}=\sup _{t \in \mathbb{R}}\left(\int_{[0,1]}\|f(t+s)\|^{p} d s\right)^{\frac{1}{p}}<\infty .
$$

The space of all such functions is denoted by $B S^{p}(\mathbb{R}, X)$ and is provided with the following norm:

$$
\begin{aligned}
\|f\|_{B S^{p}} & :=\sup _{t \in \mathbb{R}}\left(\int_{[t, t+1]}\|f(s)\|^{p} d s\right)^{\frac{1}{p}} \\
& =\sup _{t \in \mathbb{R}}\|f(t+\cdot)\|_{L^{p}([0,1], X)} .
\end{aligned}
$$

Then, the following inclusions hold:

$$
B C(\mathbb{R}, X) \subset B S^{p}(\mathbb{R}, X) \subset L_{l o c}^{p}(\mathbb{R}, X) .
$$


Definition 2.4 (Bochner transform) 11] Let $f \in L_{l o c}^{p}(\mathbb{R}, X)$ for $1 \leq p<\infty$. The Bochner transform of $f$ is the function $f^{b}: \mathbb{R} \longrightarrow L^{p}([0,1], X)$ defined for all $t \in \mathbb{R}$ by

$$
\left(f^{b}(t)\right)(s)=f(t+s) \text { for } s \in[0,1] .
$$

Now, we give the definition of almost automorphy in the sense of Stepanov.

Definition 2.5 13] Let $1 \leq p<\infty$. A function $f \in L_{l o c}^{p}(\mathbb{R}, X)$ is said to be almost automorphic in the sense of Stepanov (or $S^{p}$-almost automorphic), if for every sequence $\left(\sigma_{n}\right)_{n \geq 0}$ of real numbers, there exists a subsequence $\left(s_{n}\right)_{n \geq 0} \subset\left(\sigma_{n}\right)_{n \geq 0}$ and $g \in L_{l o c}^{p}(\mathbb{R}, X)$, such that, for each $t \in \mathbb{R}$

$$
\lim _{n}\left(\int_{t}^{t+1}\left\|f\left(s+s_{n}\right)-g(s)\right\|^{p} d s\right)^{\frac{1}{p}}=0 \quad \text { and } \quad \lim _{n}\left(\int_{t}^{t+1}\left\|g\left(s-s_{n}\right)-f(s)\right\|^{p} d s\right)^{\frac{1}{p}}=0 .
$$

The space of all such functions is denoted by $A A S^{p}(\mathbb{R}, X)$.

Theorem 2.1 13] The following are equivalent:

(i) $f$ is $S^{p}$-almost automorphic.

(ii) For every sequence $\left(\sigma_{n}\right)_{n \geq 0}$ of real numbers, there exists a subsequence $\left(s_{n}\right)_{n \geq 0} \subset\left(\sigma_{n}\right)_{n \geq 0}$ for each $t \in \mathbb{R}$

$$
\lim _{n, m}\left(\int_{t}^{t+1}\left\|f\left(\tau+s_{n}-s_{m}\right)-f(\tau)\right\|^{p} d \tau\right)^{\frac{1}{p}}=0
$$

Remark 2.2 4

(i) Every almost automorphic function is $S^{p}$-almost automorphic for $1 \leq p<\infty$.

(ii) For all $1 \leq p_{1} \leq p_{2}<\infty$, if $f$ is $S^{p_{2}}$-almost automorphic, then $f$ is $S^{p_{1}}$-almost automorphic.

(iii) The Bochner transform of an $X$-valued function is a $L^{p}([0,1], X)$-valued function. Moreover, a function $f$ is $S^{p}$-almost automorphic if and only if $f^{b}$ is (Bochner) almost automorphic.

(iv) A function $\varphi(t, s)$ for $t \in \mathbb{R}, s \in[0,1]$ is the Bochner transform of a function $f$ (i.e., $\exists$ $f: \mathbb{R} \longrightarrow X$ such that $\left.\left(f^{b}(t)\right)(s)=\varphi(t, s), t \in \mathbb{R}, s \in[0,1]\right)$ if and only if $\varphi(t+\tau, s-\tau)=\varphi(t, s)$ for all $t \in \mathbb{R}, s \in[0,1]$ and $\tau \in[s-1, s]$.

Definition 2.6 Let $1 \leq p<\infty$. A function $f: \mathbb{R} \times X \longrightarrow Y$ such that $f(\cdot, x) \in L_{l o c}^{p}(\mathbb{R}, Y)$ for each $x \in X$ is said to be almost automorphic in $t$ uniformly with respect to $x$ in $X$ if for each compact set $K$ in $X$, for every sequence $\left(\sigma_{n}\right)_{n \geq 0}$ of real numbers, there exists a subsequence $\left(s_{n}\right)_{n \geq 0} \subset\left(\sigma_{n}\right)_{n \geq 0}$ and $g(\cdot, x) \in L_{l o c}^{p}(\mathbb{R}, Y)$ for each $x \in X$, such that

$\lim _{n} \sup _{x \in K}\left(\int_{t}^{t+1}\left\|f\left(s+s_{n}, x\right)-g(s, x)\right\|^{p} d s\right)^{\frac{1}{p}}=0, \lim _{n} \sup _{x \in K}\left(\int_{t}^{t+1}\left\|g\left(s-s_{n}, x\right)-f(s, x)\right\|^{p} d s\right)^{\frac{1}{p}}=0$

are well-defined for each $t \in \mathbb{R}$.

The space of all such functions is denoted by $A A S^{p} U(\mathbb{R} \times X, Y)$.

\subsection{A subvariant minimizing functional}

The results in this section was introduced by [9] as a generalisation of the works by Fink, see [15].

Let $K$ be a compact subset of $X$. By $\mathcal{F}_{K}$, we denote the set of mild solutions $x$ on $\mathbb{R}$ of equation (1.1) with relatively compact range and by $C_{K}(\mathbb{R}, X)$ the set

$$
C_{K}(\mathbb{R}, X)=\{x \in C(\mathbb{R}, X): \quad x(t) \in K \text { for all } t \in \mathbb{R}\} .
$$

Note that $\mathcal{F}_{K} \subset C_{K}(\mathbb{R}, X)$.

Definition 2.7 A functional $\lambda_{K}: C_{K}(\mathbb{R}, X) \rightarrow \mathbb{R}$ is called a subvariant functional associated to the compact set $K$, if $\lambda_{K}$ satisfies the following conditions:

(i) $\lambda_{K}$ is invariant by translation: $\lambda_{K}\left(x_{\tau}\right)=\lambda_{K}(x)$ for each $\tau \in \mathbb{R}$, where $x_{\tau}()=.x(\tau+$.).

(ii) $\lambda_{K}$ is lower semicontinuous for the topology of compact convergence: if $\lim _{n \rightarrow+\infty} x_{n}=y$ uniformly on each compact subset of $\mathbb{R}$, then $\lambda_{K}(y) \leq \liminf _{n \rightarrow+\infty} \lambda_{K}\left(x_{n}\right)$. 
Definition 2.8 A function $x_{*}: \mathbb{R} \longrightarrow X$ is called a minimal $K$-valued solution of equation (1.1) if the following hold

$$
x_{*} \in \mathcal{F}_{K} \quad \text { and } \quad \lambda_{K}\left(x_{*}\right)=\inf _{x \in \mathcal{F}_{K}} \lambda_{K}(x)
$$

An example of subvariant functional is given by:

$$
\lambda_{K}(x)=\sup _{t \in \mathbb{R}} \Phi(x(t)) \quad \text { where } \Phi \in C(K, \mathbb{R}) .
$$

In particular, we have

$$
\lambda_{K}(x)=\sup _{t \in \mathbb{R}}\|x(t)\|
$$

\section{Almost automorphic solutions of equation (1.1)}

In this section, we prove the existence of compact almost automorphic solutions to the semilinear evolution equation (1.1).

By a mild solution of equation (1.1), we mean a continuous function $x: \mathbb{R} \longrightarrow X$ satisfying the following variation of constants formula:

$$
x(t)=T(t-\sigma) x(\sigma)+\int_{\sigma}^{t} T(t-s) f(s, x(s)) d s \quad \text { for all } t \geq \sigma .
$$

Now, we give the following hypotheses:

(H1) $(A, D(A))$ is the infinitesimal generator of a $C_{0}$-semigroup $(T(t))_{t \geq 0}$ on $X$.

(H2) $(T(t))_{t>0}$ is compact, i.e., $T(t)$ is a compact operator for each $t>0$.

(H3) For all $R>0$, the function $\sup _{\|x\| \leq R}\|f(\cdot, x)\| \in L_{l o c}^{p}(\mathbb{R}, \mathbb{R})$ such that

$$
\sup _{t \in \mathbb{R}}\left(\int_{t}^{t+1} \sup _{\|x\| \leq R}\|f(s, x)\|^{p} d s\right)^{\frac{1}{p}}<+\infty
$$

(H4) The function $f$ is given by $f(t, x):=\varphi(t) g(x)+\psi(t)$, where $\varphi \in A A S^{1}(\mathbb{R}, \mathbb{R}) \cap L^{\infty}(\mathbb{R}, \mathbb{R})$, $\psi \in A A S^{1}(\mathbb{R}, X)$ and $g \in C(X, X)$ transform bounded subsets of $X$ into bounded.

Remark 3.1 Hypothesis (H4) implies (H3).

Definition 3.1 Let $p \in[1, \infty)$ and $f: \mathbb{R} \times X \longrightarrow X$ be such that $f(\cdot, x) \in B S^{p}(\mathbb{R}, X)$ for each $x \in X$. Then, $f$ is said to be $S^{p}$-uniformly continuous with respect to the second argument on each compact $K \subset X$ if, for all $K \subset X$ compact for all $\varepsilon>0$ there exists $\delta_{K, \varepsilon}$ such that for all $x_{1}, x_{2} \in K$, we have

$$
\left\|x_{1}-x_{2}\right\| \leq \delta_{K, \varepsilon} \Longrightarrow\left(\int_{t}^{t+1}\left\|f\left(s, x_{1}\right)-f\left(s, x_{2}\right)\right\|^{p} d s\right)^{\frac{1}{p}} \leq \varepsilon \quad \text { for all } t \in \mathbb{R} .
$$

Lemma 3.1 Let $p \in[1, \infty)$ and $f: \mathbb{R} \times X \longrightarrow X$ be such that $f(\cdot, x) \in L_{l o c}^{p}(\mathbb{R}, X)$ for each $x \in X$. Then, $f \in A A S^{p} U(\mathbb{R} \times X, X)$ if and only if the following hold:

(i) For each $x \in X, f(\cdot, x) \in A A S^{p}(\mathbb{R}, X)$.

(ii) $f$ is $S^{p}$-uniformly continuous with respect to the second argument on each compact $K \subset X$.

Proof. Let $f \in A A S^{p} U(\mathbb{R} \times X, X)$ and $f^{b}: \mathbb{R} \times X \longrightarrow L^{p}([0,1], X)$ be the Bochner transform associated to $f$. It follows in view of [6. Proposition 5.5], that (i) is clearly satisfied and that: for each compact $K \subset X$, for all $\varepsilon>0$ there exists $\delta_{K, \varepsilon}$ such that for all $x_{1}, x_{2} \in K$ one has

$$
\left\|x_{1}-x_{2}\right\| \leq \delta_{K, \varepsilon} \quad \Longrightarrow \quad\left\|f^{b}\left(t, x_{1}\right)-f^{b}\left(t, x_{2}\right)\right\|_{p} \leq \varepsilon \quad \text { for all } t \in \mathbb{R} .
$$

Since

$$
\begin{aligned}
\left\|f^{b}\left(t, x_{1}\right)-f^{b}\left(t, x_{2}\right)\right\|_{p} & =\left(\int_{[0,1]}\left\|\left(f^{b}\left(t, x_{1}\right)\right)(s)-\left(f^{b}\left(t, x_{2}\right)\right)(s)\right\|^{p} d s\right)^{\frac{1}{p}} \\
& =\left(\int_{t}^{t+1}\left\|f\left(s, x_{1}\right)-f\left(s, x_{2}\right)\right\|^{p} d s\right)^{\frac{1}{p}} \quad \text { for all } t \in \mathbb{R} .
\end{aligned}
$$


It follows that (3.2) holds and then (ii) is achieved.

Conversely, let $f: \mathbb{R} \times X \longrightarrow X$ be a function such that $f(\cdot, x) \in L_{l o c}^{p}(\mathbb{R}, X)$ for each $x \in X$. Assume that $f$ satisfy (i)-(ii). Let us fix a compact subset $K$ in $X$ and $\varepsilon>0$. Since $K$ is compact, it follows that there exists a finite subset $\left\{x_{1}, \ldots, x_{n}\right\} \subset K\left(n \in \mathbb{N}^{*}\right)$ such that $K \subseteq \bigcup_{i=1}^{n} B\left(x_{i}, \delta_{K, \varepsilon}\right)$.

For $x \in K$ there exist $i=1, \ldots, n$ satisfying $\left\|x-x_{i}\right\| \leq \delta_{K, \varepsilon}$. Let $\left(\sigma_{n}\right)_{n \geq 0}$ be a sequence of real numbers and its subsequence $\left(s_{n}\right)_{n \geq 0}$ such that

$$
\begin{aligned}
& \left(\int_{t}^{t+1}\left\|f\left(s+s_{l}-s_{k}, x\right)-f(s, x)\right\|^{p} d s\right)^{\frac{1}{p}} \leq\left(\int_{t}^{t+1}\left\|f\left(s+s_{l}-s_{k}, x\right)-f\left(s+s_{l}-s_{k}, x_{i}\right)\right\|^{p} d s\right)^{\frac{1}{p}} \\
& +\left(\int_{t}^{t+1}\left\|f\left(s+s_{l}-s_{k}, x_{i}\right)-f\left(s, x_{i}\right)\right\|^{p} d s\right)^{\frac{1}{p}}+\left(\int_{t}^{t+1}\left\|f\left(s, x_{i}\right)-f(s, x)\right\|^{p} d s\right)^{\frac{1}{p}} .
\end{aligned}
$$

Using (i), it holds that $f\left(\cdot, x_{i}\right) \in A A S^{p}(\mathbb{R}, X)$. Hence, for $k, l$ large enough, for each $t \in \mathbb{R}$

$$
\left(\int_{t}^{t+1}\left\|f\left(s+s_{l}-s_{k}, x_{i}\right)-f\left(s, x_{i}\right)\right\|^{p} d s\right)^{\frac{1}{p}} \leq \frac{\varepsilon}{3} .
$$

Otherwise, since $\left\|x-x_{i}\right\| \leq \delta_{K, \delta}$ and by (ii) we claim that, for each $k, l \in \mathbb{N}$

$$
\left(\int_{t}^{t+1}\left\|f\left(s+s_{l}-s_{k}, x\right)-f\left(s+s_{l}-s_{k}, x_{i}\right)\right\|^{p} d s\right)^{\frac{1}{p}} \leq \frac{\varepsilon}{3} \quad \text { for all } t \in \mathbb{R}
$$

and

$$
\left(\int_{t}^{t+1}\left\|f(s, x)-f\left(s, x_{i}\right)\right\|^{p} d s\right)^{\frac{1}{p}} \leq \frac{\varepsilon}{3} \quad \text { for all } t \in \mathbb{R} .
$$

Consequently, if we replace (3.4), (3.5) and (3.6) in (3.3) then, for $k, l$ large enough we have

$$
\sup _{x \in K}\left(\int_{t}^{t+1}\left\|f\left(s+s_{l}-s_{k}, x\right)-f(s, x)\right\|^{p} d s\right)^{\frac{1}{p}} \leq \varepsilon .
$$

for each $t \in \mathbb{R}$.

Remark 3.2 (a) In view of Lemma 3.1. If $f$ is the function defined by (H4), then $f \in A A S^{1} U(\mathbb{R} \times$ $X, X)$.

(b) Using Lemma 3.1, we can prove easily that the function $g$ in (2.3) is $S^{p}$-uniformly continuous with respect to the second argument on each compact $K \subset X$.

Lemma 3.2 Let $f \in A A S^{p} U(\mathbb{R} \times X, X)$ for $p \in[1, \infty)$. Then, for each compact $K$ in $X$, we have (i) $k_{p}=\sup _{t \in \mathbb{R}} \sup _{x \in K}\left(\int_{t}^{t+1}\|f(s, x)\|^{p} d s\right)^{\frac{1}{p}}<+\infty$.

(ii) $l_{p}=\sup _{t \in \mathbb{R}} \sup _{x \in K}\left(\int_{t}^{t+1}\|g(s, x)\|^{p} d s\right)^{\frac{1}{p}}<+\infty$, where $g$ is the function defined in (2.3).

Proof. (i) Let $K \subset X$ be compact and let $\delta>0$. Then, there exists $\left\{x_{1}, \ldots, x_{n}\right\} \subset K\left(n \in \mathbb{N}^{*}\right)$ such that $K \subseteq \bigcup_{i=1}^{n} B\left(x_{i}, \delta\right)$. Therefore, for each $x \in K$ there exist $i=1, \ldots, n$ satisfying $\left\|x-x_{i}\right\| \leq \delta$. Then, for all $x \in K$ and $\varepsilon>0$, we have

$$
\left(\int_{t}^{t+1}\|f(s, x)\|^{p} d s\right)^{\frac{1}{p}} \leq\left(\int_{t}^{t+1}\left\|f(s, x)-f\left(s, x_{i}\right)\right\|^{p} d s\right)^{\frac{1}{p}}+\sum_{i=1}^{n}\left(\int_{t}^{t+1}\left\|f\left(s, x_{i}\right)\right\|^{p} d s\right)^{\frac{1}{p}}
$$

for all $t \in \mathbb{R}$. Hence, by Lemma 3.1 we claim that

$$
\sup _{t \in \mathbb{R}} \sup _{x \in K}\left(\int_{t}^{t+1}\|f(s, x)\|^{p} d s\right)^{\frac{1}{p}} \leq \varepsilon+\sum_{i=1}^{n} \sup _{t \in \mathbb{R}}\left(\int_{t}^{t+1}\left\|f\left(s, x_{i}\right)\right\|^{p} d s\right)^{\frac{1}{p}}<\infty .
$$


(ii) Let $K \subset X$ be compact and let $\left(\sigma_{n}\right)_{n \geq 0}$ be a sequence. Since $f \in A A S^{p} U(\mathbb{R} \times X, X)$, it follows that there exists a subsequence $\left(s_{n}\right)_{n \geq 0} \subset\left(\sigma_{n}\right)_{n \geq 0}$ such that (2.3) holds. Hence, for all $n \in \mathbb{N}$

$$
\begin{aligned}
\left(\int_{t}^{t+1}\|g(s, x)\|^{p} d s\right)^{\frac{1}{p}} \leq & \sup _{x \in K}\left(\int_{t}^{t+1}\left\|f\left(s+s_{n}, x\right)-g(s, x)\right\|^{p} d s\right)^{\frac{1}{p}} \\
& +\sup _{x \in K}\left(\int_{t}^{t+1}\left\|f\left(s+s_{n}, x\right)\right\|^{p} d s\right)^{\frac{1}{p}} \\
\leq & \sup _{x \in K}\left(\int_{t}^{t+1}\left\|f\left(s+s_{n}, x\right)-g(s, x)\right\|^{p} d s\right)^{\frac{1}{p}}+k_{p} \text { for all } x \in K, t \in \mathbb{R} .
\end{aligned}
$$

Therefore, for $n$ large enough, we have

$$
\sup _{x \in K}\left(\int_{t}^{t+1}\|g(s, x)\|^{p} d s\right)^{\frac{1}{p}} \leq k_{p} \quad \text { for all } t \in \mathbb{R} .
$$

This proves the result.

Lemma 3.3 [3, Theorem 4.1.2] Let $Y$ be a normed space and $\left(T_{i}\right)_{i \in T}$ be any family of bounded linear operators on $Y$ such that $\sup _{i \in I}\left|T_{i}\right|<\infty$. If $D$ is a dense subset of $Y$, and if for each $y \in D$

$$
T_{i} y \rightarrow T y \text { as } i \rightarrow \infty,
$$

for some bounded linear operator $T$. Then for every compact set $K \subset Y$

$$
\sup _{y \in K}\left\|T_{i} y-T y\right\| \rightarrow 0 \text { as } i \rightarrow \infty .
$$

The following Lemma is needed in the proof of Theorem 3.1 .

Lemma 3.4 Let $\left(x_{n}\right)_{n}$ be a sequence in a Banach space $X$ and $x \in X$. If every subsequence $\left(x_{n}^{\prime}\right)_{n} \subset\left(x_{n}\right)_{n}$ has a subsequence $\left(x_{n}^{\prime \prime}\right)_{n} \subset\left(x_{n}^{\prime}\right)_{n} \subset\left(x_{n}\right)_{n}$ that converges to $x$, then the whole sequence $\left(x_{n}\right)_{n}$ converges to $x$.

Proof. By contradiction.

Theorem 3.1 Let $f \in A A S^{p} U(\mathbb{R} \times X, X)$ for $p \in[1, \infty)$. Assume that (H1) holds. Then, a mild solution of equation (1.1) with relatively compact range is uniformly continuous.

Proof. Let $x$ be a solution of equation (1.1) such that $K=\overline{\{x(t): t \in \mathbb{R}\}} \subset X$ is compact. We distinguish two cases:

The case $p \in(1, \infty)$. Since $(T(t))_{t \geq 0}$ is a $C_{0}$-semigroup on $X$, it follows in view of Lemma 3.3 , that

$$
\lim _{t \rightarrow 0} \sup _{x \in K}\|T(t) x-x\|=0 .
$$

Furthermore, the $C_{0}$-semigroup $(T(t))_{t \geq 0}$ on $X$ satisfies

$$
\|T(t)\| \leq M e^{\omega t} \quad \text { for } t \geq 0
$$

for some $M \geq 1, \omega \in \mathbb{R}$. Let $q$ be such that $\frac{1}{p}+\frac{1}{q}=1$. Using Hölder inequality, we have for all $t, s \in \mathbb{R}$,

$$
\begin{aligned}
\|x(t)-x(s)\| & \leq\|T(t-s) x(s)-x(s)\|+\int_{s}^{t}\|T(t-\sigma) f(\sigma, x(\sigma))\| d \sigma \\
& \leq \sup _{x \in K}\|T(t-s) x-x\|+M\left(\int_{s}^{t} e^{q \omega(t-\sigma)} d \sigma\right)^{\frac{1}{q}}\left(\int_{s}^{t}\|f(\sigma, x(\sigma))\|^{p} d \sigma\right)^{\frac{1}{p}} \\
& \leq \sup _{x \in K}\|T(t-s) x-x\|+k_{p} M(t-s+3)^{\frac{1}{p}}\left(\int_{0}^{t-s} e^{q \omega \sigma} d \sigma\right)^{\frac{1}{q}}, \quad t \geq s .
\end{aligned}
$$


Then, by interchanging $s$ and $t$, we claim that

$$
\|x(t)-x(s)\| \leq \sup _{x \in K}\|T(|t-s|) x-x\|+k_{p} M^{\prime}(|t-s|+3)^{\frac{1}{p}}\left(\int_{0}^{|t-s|} e^{q \omega \sigma} d \sigma\right)^{\frac{1}{q}} .
$$

From $\left(\int_{0}^{|t-s|} e^{q \omega \sigma} d \sigma\right)^{\frac{1}{q}} \rightarrow 0$ as $|t-s| \rightarrow 0$ and (3.7) we deduce that

$$
\|x(t)-x(s)\| \rightarrow 0 \text { as }|t-s| \rightarrow 0
$$

Consequently, $x$ is uniformly continuous.

The case $p=1$. To show that $x$ is uniformly continuous, we take two real sequences $\left(t_{n}\right)_{n}$ and $\left(s_{n}\right)_{n}$ such that $\left|t_{n}-s_{n}\right| \rightarrow 0$ as $n \rightarrow \infty$ and we prove that $y_{n}=x\left(t_{n}\right)-x\left(s_{n}\right) \rightarrow 0$ as $n \rightarrow \infty$. In fact, consider the sequence $\left(h_{n}\right)_{n}$ defined by $h_{n}=t_{n}-s_{n}$. Then, we have $\lim _{n \rightarrow \infty} h_{n}=0$. Assume without loss of generality that $0 \leq h_{n} \leq 1$ for all $n \in \mathbb{N}$. Thus, we have

$$
\begin{aligned}
x\left(t_{n}\right)=x\left(s_{n}+h_{n}\right) & =T\left(h_{n}\right) x\left(s_{n}\right)+\int_{s_{n}}^{s_{n}+h_{n}} T\left(s_{n}+h_{n}-s\right) f(s, x(s)) d s \\
& =T\left(h_{n}\right) x\left(s_{n}\right)+\int_{0}^{h_{n}} T\left(h_{n}-s\right) f\left(s+s_{n}, x\left(s+s_{n}\right)\right) d s .
\end{aligned}
$$

Let $M \geq 1$ and $\omega \in \mathbb{R}$ such that $\|T(t)\| \leq M e^{\omega t}$ for all $t \geq 0$. Then

$$
\begin{aligned}
\left\|x\left(s_{n}+h_{n}\right)-x\left(s_{n}\right)\right\| & \leq\left\|T\left(h_{n}\right) x\left(s_{n}\right)-x\left(s_{n}\right)\right\|+\int_{0}^{h_{n}}\left\|T\left(h_{n}-s\right) f\left(s+s_{n}, x\left(s+s_{n}\right)\right)\right\| d s \\
& \leq \sup _{y \in K}\left\|T\left(h_{n}\right) y-y\right\|+M \int_{0}^{h_{n}} e^{\omega\left(h_{n}-s\right)}\left\|f\left(s+s_{n}, x\left(s+s_{n}\right)\right)\right\| d s \\
& \leq \sup _{y \in K}\left\|T\left(h_{n}\right) y-y\right\|+M e^{|\omega| h_{n}} \int_{0}^{h_{n}}\left\|f\left(s+s_{n}, x\left(s+s_{n}\right)\right)\right\| d s .
\end{aligned}
$$

The semigroup $(T(t))_{t \geq 0}$ is strongly continuous, then for each $y \in K, T\left(h_{n}\right) y \rightarrow y$ as, $n \rightarrow \infty$. This implies that $\sup _{n}\left\|T\left(h_{n}\right) y\right\|<\infty$ for each $y \in X$ and thus by the Banach-Steinhaus's Theorem $\sup _{n}\left\|T\left(h_{n}\right)\right\|<\infty$. It follows from Lemma 3.3 that

$$
\sup _{y \in K}\left\|T\left(h_{n}\right) y-y\right\| \rightarrow 0 \quad \text { as } n \rightarrow \infty .
$$

Let $y_{n}^{\prime}=x\left(s_{n}^{\prime}+h_{n}^{\prime}\right)-x\left(s_{n}^{\prime}\right)$ be a subsequence of $y_{n}$. From the $S^{1}$-almost automorphy of $f$, there exist a subsequence $\left(s_{n}^{\prime \prime}\right)_{n} \subset\left(s_{n}^{\prime}\right)_{n}$ and there exist $g: \mathbb{R} \times X \rightarrow X$ with $g(\cdot, x) \in L_{l o c}^{1}(\mathbb{R}, X)$ for each $x \in X$ such that for every $t \in \mathbb{R}$, we have

$$
\sup _{x \in K} \int_{t}^{t+1}\left\|f\left(s+s_{n}^{\prime \prime}, x\right)-g(s, x)\right\| d s \rightarrow 0 \text { as } n \rightarrow \infty .
$$

Let $\left(h_{n}^{\prime \prime}\right)_{n}$ be the corresponding subsequence of $\left(h_{n}^{\prime}\right)_{n}$. We can assume that $0 \leq h_{n} \leq 1$ for all $n \in \mathbb{N}$. Then, we have

$$
\begin{aligned}
\int_{0}^{h_{n}^{\prime \prime}}\left\|f\left(s+s_{n}^{\prime \prime}, x\left(s+s_{n}^{\prime \prime}\right)\right)\right\| d s & \leq \int_{0}^{h_{n}^{\prime \prime}}\left\|f\left(s+s_{n}^{\prime \prime}, x\left(s+s_{n}^{\prime \prime}\right)\right)-g\left(s, x\left(s+s_{n}^{\prime \prime}\right)\right)\right\| d s \\
& +\int_{0}^{h_{n}^{\prime \prime}}\left\|g\left(s, x\left(s+s_{n}^{\prime \prime}\right)\right)\right\| d s \\
& \leq \sup _{x \in K} \int_{0}^{h_{n}^{\prime \prime}}\left\|f\left(s+s_{n}^{\prime \prime}, x\right)-g(s, x)\right\| d s+\int_{0}^{h_{n}^{\prime \prime}}\left\|g\left(s, x\left(s+s_{n}^{\prime \prime}\right)\right)\right\| d s \\
& \leq \sup _{x \in K} \int_{0}^{1}\left\|f\left(s+s_{n}^{\prime \prime}, x\right)-g(s, x)\right\| d s+\int_{0}^{h_{n}^{\prime \prime}}\left\|g\left(s, x\left(s+s_{n}^{\prime \prime}\right)\right)\right\| d s .
\end{aligned}
$$

Using (3.10) we claim that $\lim _{n \rightarrow \infty} \sup _{x \in K} \int_{0}^{1}\left\|f\left(s+s_{n}^{\prime \prime}, x\right)-g(s, x)\right\| d s=0$.

On the other hand, let $\delta>0$. Then, there exist $x_{1}, \ldots, x_{m} \in K, m \in \mathbb{N}^{*}$, such that , for each 
$x \in K$ there exists $i_{0}=1, \ldots, m$ satisfies $\left\|x-x_{i_{0}}\right\| \leq \delta$. Let $\varepsilon>0$, then, for all $s \in \mathbb{R}$ for all $n \in \mathbb{N}$, there exists $i(s, n)=1, \ldots, m$ with $\left\|x\left(s+s_{n}^{\prime \prime}\right)-x_{i(s, n)}\right\| \leq \delta$. Hence, by Remark 3.2 we obtain

$$
\begin{aligned}
\int_{0}^{h_{n}^{\prime \prime}}\left\|g\left(s, x\left(s+s_{n}^{\prime \prime}\right)\right)\right\| d s & \leq \int_{0}^{1}\left\|g\left(s, x\left(s+s_{n}^{\prime \prime}\right)\right)-g\left(s, x_{i(s, n)}\right)\right\| d s+\sum_{i=1}^{m} \int_{0}^{h_{n}^{\prime \prime}}\left\|g\left(s, x_{i}\right)\right\| d s \\
& \leq \varepsilon+\sum_{i=1}^{m} \int_{0}^{h_{n}^{\prime \prime}}\left\|g\left(s, x_{i}\right)\right\| d s \quad \text { for all } \varepsilon>0 .
\end{aligned}
$$

By Lemma 3.2, we claim that, for each $i=1, \ldots, m$, we have

$$
\int_{0}^{h_{n}^{\prime \prime}}\left\|g\left(s, x_{i}\right)\right\| d s \rightarrow 0 \quad \text { as } n \rightarrow \infty
$$

Then, $\int_{0}^{h_{n}^{\prime \prime}}\left\|g\left(s, x\left(s+s_{n}^{\prime \prime}\right)\right)\right\| d s \rightarrow 0$ as $n \rightarrow \infty$. Therefore,

$$
\int_{0}^{h_{n}^{\prime \prime}}\left\|f\left(s+s_{n}^{\prime \prime}, x\left(s+s_{n}^{\prime \prime}\right)\right)\right\| d s \rightarrow 0 \quad \text { as } n \rightarrow \infty .
$$

Consequently, we conclude from (3.8), (3.9) and (3.11) that

$$
\left\|y_{n}^{\prime \prime}\right\|=\left\|x\left(t_{n}^{\prime \prime}\right)-x\left(s_{n}^{\prime \prime}\right)\right\|=\left\|x\left(s_{n}^{\prime \prime}+h_{n}^{\prime \prime}\right)-x\left(s_{n}^{\prime \prime}\right)\right\| \rightarrow 0 \quad \text { as } n \rightarrow \infty,
$$

Finally, using Lemma 3.4 the whole sequence $y_{n}=x\left(t_{n}\right)-x\left(s_{n}\right)$ converges to 0 . We conclude that the mild solution $x$ is uniformly continuous.

Lemma 3.5 Let $f \in A A S^{p}(\mathbb{R} \times X, X)$ for $p \in[1, \infty)$. Assume that (H1)-(H2) hold. If equation (1.1) has at least a mild solution $x_{0}:\left[t_{0},+\infty\right) \longrightarrow X$ with relatively compact range. Then, there exists a mild solution $x$ on $\mathbb{R}$ of equation (1.1) such that

$$
\{x(t): t \in \mathbb{R}\} \subset \overline{\left\{x_{0}(t): t \geq t_{0}\right\}} .
$$

Proof. Let $K:=\overline{\left\{x_{0}(t): t \geq t_{0}\right\}} \subset X$. By assumption $K$ is compact. Moreover, the mild solution $x_{0}$ is given by:

$$
x_{0}(t)=T(t-s) x_{0}(s)+\int_{s}^{t} T(t-\sigma) f\left(\sigma, x_{0}(\sigma)\right) d \sigma \quad \text { for } t \geq s \geq t_{0} .
$$

Let $\left(t_{n}^{\prime}\right)_{n}$ be a sequence of real numbers such that

$$
\lim _{n \rightarrow+\infty} t_{n}^{\prime}=+\infty
$$

Since $f \in A A S^{p} U(\mathbb{R} \times X, X)$, it follows that there exist a subsequence $\left(t_{n}\right)_{n} \subset\left(t_{n}^{\prime}\right)_{n}$ and $g$ : $\mathbb{R} \times K \rightarrow X$ such that for each $t \in \mathbb{R}$, we have

$$
\lim _{n} \sup _{x \in K}\left(\int_{t}^{t+1}\left\|f\left(s+s_{n}, x\right)-g(s, x)\right\|^{p} d s\right)^{\frac{1}{p}}=0 .
$$

For $n \in \mathbb{N}$ sufficiently large, the function $t \mapsto x_{0}\left(\cdot+t_{n}\right) \in K$ is defined on $\mathbb{R}$. Let $u_{n}(t):=x_{0}\left(t+t_{n}\right)$ for all $t \in \mathbb{R}$ and the set $U_{n}=\left\{u_{n}: n \in \mathbb{N}\right\}$. Then, $U_{n} \subset C(\mathbb{R}, X)$ and $\left(u_{n}\right)_{n}$ satisfies for each $t \in \mathbb{R} u_{n}(t) \in K$. Therefore, for each $t \in \mathbb{R}$, the subset $U_{n}(t)=\left\{u_{n}(t): n \in \mathbb{N}\right\}$ is relatively compact in $X$. By Theorem 3.1 and using Arzela-Ascoli's Theorem and an extraction diagonal argument applied to the sequence $\left(u_{n}\right)_{n}$, we claim that there exist $x_{*} \in C(\mathbb{R}, X)$ and a subsequence of $\left(t_{n}\right)_{n}$ such that

$$
x_{0}\left(t+t_{n}\right) \rightarrow x_{*}(t) \quad \text { as } \quad n \rightarrow+\infty,
$$

uniformly on each compact subset of $\mathbb{R}$. Hence, for $n \in \mathbb{N}$ sufficiently large, we have

$$
x_{0}\left(t+t_{n}\right)=T(t-s) x_{0}\left(s+t_{n}\right)+\int_{s}^{t} T(t-\sigma) f\left(\sigma+t_{n}, x_{0}\left(\sigma+t_{n}\right)\right) d \sigma, \quad t \geq s .
$$


Let $t, s \in \mathbb{R}, t \geq s$. Then, using (3.14), we claim that

$$
\lim _{n \rightarrow+\infty} T(t-s) x_{0}\left(s+t_{n}\right)=T(t-s) x_{*}(s) .
$$

Now, we prove that

$$
\lim _{n \rightarrow+\infty} \int_{s}^{t} T(t-\sigma) f\left(\sigma+t_{n}, x_{0}\left(\sigma+t_{n}\right)\right) d \sigma=\int_{s}^{t} T(t-\sigma) g\left(\sigma, x_{*}(\sigma)\right) d \sigma .
$$

In fact, we have

$$
\begin{aligned}
& \left(\int_{t}^{t+1}\left\|f\left(\sigma+t_{n}, x_{0}\left(\sigma+t_{n}\right)\right)-g\left(\sigma, x_{*}(\sigma)\right)\right\|^{p} d \sigma\right)^{\frac{1}{p}} \\
\leq & \left(\int_{t}^{t+1}\left\|f\left(\sigma+t_{n}, x_{0}\left(\sigma+t_{n}\right)\right)-g\left(\sigma, x_{0}\left(\sigma+t_{n}\right)\right)\right\|^{p} d \sigma\right)^{\frac{1}{p}} \\
+ & \left(\int_{t}^{t+1}\left\|g\left(\sigma, x_{0}\left(\sigma+t_{n}\right)\right)-g\left(\sigma, x_{*}(\sigma)\right)\right\|^{p} d \sigma\right)^{\frac{1}{p}} \\
\leq & \underbrace{\sup _{x \in K}\left(\int_{t}^{t+1}\left\|f\left(\sigma+t_{n}, x\right)-g(\sigma, x)\right\|^{p} d \sigma\right)^{\frac{1}{p}}}_{(\mathbf{I})} \\
+ & \underbrace{\left(\int_{t}^{t+1}\left\|g\left(\sigma, x_{0}\left(\sigma+t_{n}\right)\right)-g\left(\sigma, x_{*}(\sigma)\right)\right\|^{p} d \sigma\right)^{\frac{1}{p}}}_{(\mathbf{I I})}, t \in \mathbb{R} .
\end{aligned}
$$

Using (3.13), we obtain that $(\mathbf{I}) \rightarrow 0$ as $n \rightarrow \infty$. On the other hand, by Remark 3.2. it follows that $g$ is $S^{p}$-uniformly continuous. Therefore, we have (II) $\rightarrow 0$ as $n \rightarrow \infty$. Consequently, for each $t \in \mathbb{R}$

$$
K_{p}(t, n):=\left(\int_{t}^{t+1}\left\|f\left(\sigma+t_{n}, x_{0}\left(\sigma+t_{n}\right)\right)-g\left(\sigma, x_{*}(\sigma)\right)\right\|^{p} d \sigma\right)^{\frac{1}{p}} \rightarrow 0 \text { as } n \rightarrow \infty .
$$

Thus, for $p \in[1, \infty)$, we have

$$
\begin{aligned}
& \left\|\int_{s}^{t} T(t-\sigma) f\left(\sigma+t_{n}, x_{0}\left(\sigma+t_{n}\right)\right) d \sigma-\int_{s}^{t} T(t-\sigma) g\left(\sigma, x_{*}(\sigma)\right) d \sigma\right\| \\
\leq & \int_{s}^{t}\left\|T(t-\sigma)\left[f\left(\sigma+t_{n}, x_{0}\left(\sigma+t_{n}\right)\right)-g\left(\sigma, x_{*}(\sigma)\right)\right]\right\| d \sigma \\
\leq & M \sum_{m=[s]}^{[t]+1} e^{|\omega|(t-m)} \int_{m}^{m+1}\left\|f\left(\sigma+t_{n}, x_{0}\left(\sigma+t_{n}\right)\right)-g\left(\sigma, x_{*}(\sigma)\right)\right\| d \sigma \\
\leq & M \sum_{m=[s]}^{[t]+1} e^{|\omega|(t-m)}\left(\int_{m}^{m+1}\left\|f\left(\sigma+t_{n}, x_{0}\left(\sigma+t_{n}\right)\right)-g\left(\sigma, x_{*}(\sigma)\right)\right\|^{p} d \sigma\right)^{\frac{1}{p}} \\
= & M \sum_{m=[s]}^{[t]+1} e^{|\omega|(t-m)} k_{p}(m, n)
\end{aligned}
$$

Therefore, using the limit in (3.18) we obtain (3.17). Consequentely, by (3.16) and (3.17) we deduce that

$$
x_{*}(t)=T(t-s) x_{*}(s)+\int_{s}^{t} T(t-\sigma) g\left(\sigma, x_{*}(\sigma)\right) d \sigma \quad \text { for } t \geq s .
$$

Hence, $x_{*}$ is a mild solution with a relatively compact range of the following equation

$$
x_{*}^{\prime}(t)=A x_{*}(t)+g\left(t, x_{*}(t)\right), \quad t \in \mathbb{R} .
$$

By taking the sequence $\left(-t_{n}\right)_{n}$ instead of $\left(t_{n}\right)_{n}$, we apply the same construction to the mild solution $x_{*}$ of equation (3.19). Then, we obtain the existence of a mild solution $x$ with relatively compact range of equation (1.1). Consequently, $x$ is a mild solution satisfying (3.12). 


\subsection{Solutions with relatively compact ranges}

In this section, we investigate the relative compactness of the range of bounded solutions to equation (1.1) on the right half-line. We distinguish, two cases, $p \in(1, \infty)$ and $p=1$.

To conclude our results, we use the Kuratowski measure of noncompactness $\alpha$ of bounded subsets $B$ in $X$ defined by:

$$
\alpha(B):=\inf \{\varepsilon>0: B \text { has a finite cover of balls with diameter }<\varepsilon\} .
$$

The measure $\alpha$ satisfies the following properties:

(a) $\alpha(B)=0 \Leftrightarrow B$ is relatively compact.

(b) $\alpha\left(B_{1}+B_{2}\right) \leq \alpha\left(B_{1}\right)+\alpha\left(B_{2}\right)$ for all $B_{1}$ and $B_{2}$ bounded in $X$.

(c) $\alpha(B(0, \varepsilon)=2 \varepsilon$ for all $\varepsilon>0$.

For more details, we refer to 19 .

The case $p \in(1, \infty)$.

Lemma 3.6 Assume that (H1)-(H3) hold. If equation (1.1) has at least a bounded mild solution $x_{0}:\left[t_{0},+\infty\right) \longrightarrow X$. Then, its range $\left\{x_{0}(t): t \geq t_{0}\right\}$ is relatively compact in $X$.

Proof. (i) For $0<\varepsilon<1$, define

$$
x_{0}(t)=T(\varepsilon) x_{0}(t-\varepsilon)+\int_{t-\varepsilon}^{t} T(t-\sigma) f\left(\sigma, x_{0}(\sigma)\right) d \sigma \quad \text { for } t>t_{0}+1 .
$$

Let $t>t_{0}+1$. Then, we have

$$
T(\varepsilon) x_{0}(t-\varepsilon) \in T(\varepsilon) \bar{B}\left(0, M_{0}\right)
$$

where $M_{0}:=\sup _{t \geq t_{0}}\left\|x_{0}(t)\right\|<+\infty$ and $\bar{B}\left(0, M_{0}\right)$ is the closed ball of center 0 and radius $M_{0}$. Using Hölder inequality, we obtain

$\left\|\int_{t-\varepsilon}^{t} T(t-\sigma) f\left(\sigma, x_{0}(\sigma)\right) d \sigma\right\| \leq M e^{|\omega| \varepsilon} \varepsilon^{\frac{1}{q}}\left(\int_{t-\varepsilon}^{t}\left\|f\left(\sigma, x_{0}(\sigma)\right)\right\|^{p} d \sigma\right)^{\frac{1}{p}} \leq M e^{|\omega| \varepsilon} \varepsilon^{\frac{1}{q}} \tilde{k}_{p}:=\delta(\varepsilon)$,

where $\tilde{k}_{p}=\sup _{t \in \mathbb{R}}\left(\int_{t-1}^{t} \sup _{x \in \bar{B}\left(0, M_{0}\right)}\|f(\sigma, x)\|^{p} d \sigma\right)^{\frac{1}{p}}$.

Hence,

$$
\int_{t-\varepsilon}^{t} T(t-\sigma) f\left(\sigma, x_{0}(\sigma)\right) d \sigma \in \bar{B}(0, \delta(\varepsilon))
$$

Consequently,

$$
\left\{x_{0}(t): t \geq t_{0}\right\} \subset\left\{x_{0}(t): t \in\left[t_{0}, t_{0}+1\right]\right\} \cup T(\varepsilon) \bar{B}\left(0, M_{0}\right) \cup \bar{B}(0, \delta(\varepsilon)) .
$$

Thus, by the continuity of $x_{0}$, the set $\left\{x_{0}(t): t \in\left[t_{0}, t_{0}+1\right]\right\}$ is compact. Hence

$$
\alpha\left(\left\{x_{0}(t): t \in\left[t_{0}, t_{0}+1\right]\right\}\right)=0 .
$$

Therefore, from hypothesis (H2), we claim that

$$
\alpha\left(\left\{x_{0}(t): t \geq t_{0}\right\}\right) \leq 2 \delta(\varepsilon) \text { for all } \varepsilon>0 .
$$

Since $\delta(\varepsilon) \rightarrow 0$ as $\varepsilon \rightarrow 0$. It follows that

$$
\alpha\left(\left\{x_{0}(t): t \geq t_{0}\right\}\right)=0 .
$$

The case $p=1$.

In this case, the following Lemmas are needed. 
Lemma 3.7 Let $\rho \in A A S^{1}(\mathbb{R}, X)$. Then, for all $0<\varepsilon<1$, the following function:

$$
x_{\rho}^{\varepsilon}(t):=\int_{t-\varepsilon}^{t} T(t-s) \rho(s) d s \quad \text { for } t \geq t_{0}, t_{0} \in \mathbb{R}
$$

has a relatively compact range on $\left[t_{0}, \infty\right)$.

Proof. Let $0<\varepsilon<1$ and $\left(t_{n}^{\prime}\right)_{n} \subset \mathbb{R}$ be a sequence. Since $\rho \in A A S^{1}(\mathbb{R}, X)$ it follows that, there exist a subsequence $\left(t_{n}\right)_{n} \subset\left(t_{n}^{\prime}\right)_{n}$ and $g \in L_{l o c}^{1}(\mathbb{R}, X)$ such that for each $t \in \mathbb{R}$

$$
\int_{t}^{t+1}\left\|\rho\left(t_{n}+s\right)-g(s)\right\| d s \rightarrow 0 \text { as } n \rightarrow \infty .
$$

Let $y_{\rho}^{\varepsilon}:=\int_{0}^{\varepsilon} T(\sigma) g(-\sigma) d \sigma \in X$. Then,

$$
\begin{aligned}
\left\|x_{\rho}^{\varepsilon}\left(t_{n}\right)-y_{\rho}^{\varepsilon}\right\| & =\left\|\int_{t_{n}}^{t_{n}-\varepsilon} T\left(t_{n}-s\right) \rho(s) d s-\int_{0}^{\varepsilon} T(\sigma) g(-\sigma) d \sigma\right\| \\
& =\left\|\int_{0}^{\varepsilon} T(\sigma)\left[\rho\left(t_{n}-\sigma\right)-g(-\sigma)\right] d \sigma\right\| \\
& \leq M e^{|\omega|} \int_{0}^{1}\left\|\rho\left(t_{n}-\sigma\right)-g(-\sigma)\right\| d \sigma .
\end{aligned}
$$

Using (3.23), we obtain that

$$
\int_{0}^{1}\left\|\rho\left(t_{n}-\sigma\right)-g(-\sigma)\right\| d s \rightarrow 0 \text { as } n \rightarrow \infty
$$

independently of $t$ and $\varepsilon$. Therefore

$$
x_{\rho}^{\varepsilon}\left(t_{n}\right) \rightarrow y_{\rho}^{\varepsilon} \text { as } n \rightarrow \infty .
$$

Consequently, $x_{\rho}^{\varepsilon}$ has a relatively compact range on $\left[t_{0}, \infty\right)$.

Lemma 3.8 Let $f: \mathbb{R} \times X \longrightarrow X$ satisfy (H4) with $\phi, \psi \in A A S^{1}(\mathbb{R}, X)$. Assume that (H1)(H2) hold. If equation (1.1) has at least a bounded mild solution $x_{0}:\left[t_{0},+\infty\right) \longrightarrow X$, then $x_{0}$ has a relatively compact range.

Proof. Let $0<\varepsilon<1$. Define

$$
x_{0}(t)=T(\varepsilon) x_{0}(t-\varepsilon)+\int_{t-\varepsilon}^{t} T(t-\sigma) f\left(\sigma, x_{0}(\sigma)\right) d \sigma \quad \text { for } t>t_{0}+1 .
$$

Let $t>t_{0}+1$. Then, we have

$$
T(\varepsilon) x_{0}(t-\varepsilon) \in T(\varepsilon) \bar{B}\left(0, M_{0}\right)
$$

where $M_{0}:=\sup _{t \geq t_{0}}\left\|x_{0}(t)\right\|<+\infty$ and $\bar{B}\left(0, M_{0}\right)$ is the closed ball of center 0 and radius $M_{0}$. It suffices to prove that the measure of noncompactness $\alpha$ of $\int_{t-\varepsilon}^{t} T(t-\sigma) f\left(\sigma, x_{0}(\sigma)\right) d \sigma$ equals zero. In fact, by assumption on $f$, we obtain that

$$
\begin{gathered}
\int_{t-\varepsilon}^{t} T(t-\sigma) f\left(\sigma, x_{0}(\sigma)\right) d \sigma=\int_{t-\varepsilon}^{t} T(t-\sigma) \phi(\sigma) g\left(x_{0}(\sigma)\right) d \sigma+\int_{t-\varepsilon}^{t} T(t-\sigma) \psi(\sigma) d \sigma \\
\quad\left\|\int_{t-\varepsilon}^{t} T(t-\sigma) \phi(\sigma) g\left(x_{0}(\sigma)\right) d \sigma\right\| \leq M e^{|\omega| \varepsilon}|\phi|_{\infty} \sup _{x \in \bar{B}\left(0, M_{0}\right)}\|g(x)\| \varepsilon:=\gamma(\varepsilon) .
\end{gathered}
$$

Hence,

$$
\int_{t-\varepsilon}^{t} T(t-\sigma) f\left(\sigma, x_{0}(\sigma)\right) d \sigma \in \bar{B}(0, \gamma(\varepsilon))+\left\{x_{\psi}^{\varepsilon}(t): t>t_{0}+1\right\} .
$$

By Lemma 3.7 and (H2), it follows that

$$
\alpha\left(\left\{x_{0}(t): t \geq t_{0}\right\}\right) \leq 2 \gamma(\varepsilon) .
$$

Since $\gamma(\varepsilon) \rightarrow 0$ as $\varepsilon \rightarrow 0$. We claim that

$$
\alpha\left(\left\{x_{0}(t): t \geq t_{0}\right\}\right)=0 .
$$


In general when $f$ does not satisfy (H4), Lemma 3.8 cannot hold. However, under the additional assumption (H5) below, we have the following main result which is of independent interest.

(H5) For all bounded subset $B \subset X$, the set $\Gamma:=\{f(t, x):(t, x) \in \mathbb{R} \times X\}$ is relatively compact in $X$.

Proposition 3.1 Let $f: \mathbb{R} \times X \longrightarrow X$ such that $f(\cdot, x) \in B S^{1}(\mathbb{R}, X)$ for each $x \in X$. Assume that (H1)-(H2) and (H5) are satisfied. If equation (1.1) has at least a bounded mild solution $x_{0}:\left[t_{0},+\infty\right) \longrightarrow X$, then, its range is relatively compact in $X$.

Proof. Let $0<\varepsilon<1$. The mild solution $x_{0}$ satisfies the following:

$$
x_{0}(t)=T(\varepsilon) x_{0}(t-\varepsilon)+\int_{t-\varepsilon}^{t} T(t-\sigma) f\left(\sigma, x_{0}(\sigma)\right) d \sigma \quad \text { for } t \geq t_{0} .
$$

Let $x_{0}^{\varepsilon}(t):=\int_{t-\varepsilon}^{t} T(t-\sigma) f\left(\sigma, x_{0}(\sigma)\right) d \sigma$. As in the proof of Lemma 3.8 it remins to prove that, the set

$$
\left\{x_{0}^{\varepsilon}(t): t>t_{0}+1\right\}
$$

is relatively compact in $X$. In fact, let $\left(t_{n}^{\prime}\right)_{n} \subset \mathbb{R}$ and for each $t \in \mathbb{R}$, we define the sequence $\left(f\left(t_{n}^{\prime}-t, x_{0}\left(t_{n}^{\prime}-t\right)\right)\right)_{n}$. Using (H5), there exist as subsequence $\left(t_{n}\right)_{n} \subset\left(t_{n}^{\prime}\right)_{n}$ and $g: \mathbb{R} \longrightarrow X$ measurable such that for each $t \in \mathbb{R}$

$$
f\left(t_{n}-t, x_{0}\left(t_{n}-t\right)\right) \rightarrow g(t) \text { as } n \rightarrow \infty .
$$

Given $y_{0}^{\varepsilon}:=\int_{0}^{\varepsilon} T(\sigma) g(\sigma) d \sigma$. Then

$$
\begin{aligned}
\left\|x_{0}^{\varepsilon}\left(t_{n}\right)-y_{0}^{\varepsilon}\right\| & =\left\|\int_{t_{n}}^{t_{n}-\varepsilon} T\left(t_{n}-s\right) f\left(s, x_{0}(s)\right) d s-\int_{0}^{\varepsilon} T(\sigma) g(\sigma) d \sigma\right\| \\
& \leq M e^{|\omega| \varepsilon} \int_{0}^{\varepsilon}\left\|f\left(t_{n}-\sigma, x_{0}\left(t_{n}-\sigma\right)\right)-g(\sigma)\right\| d \sigma .
\end{aligned}
$$

Hence, using (3.25), it follows in view of dominated convergence Theorem that

$$
\int_{0}^{\varepsilon}\left\|f\left(t_{n}-\sigma, x_{0}\left(t_{n}-\sigma\right)\right)-g(\sigma)\right\| d \sigma \rightarrow 0 \text { as } n \rightarrow \infty,
$$

which implies that

$$
\left\|x_{0}^{\varepsilon}\left(t_{n}\right)-y_{0}^{\varepsilon}\right\| \rightarrow 0 \text { as } n \rightarrow \infty
$$

Therefore,

$$
\alpha\left(\left\{x_{0}^{\varepsilon}(t): t>t_{0}+1\right\}\right) .
$$

Consequently, using hypothesis (H2), we obtain that

$$
\alpha\left(\left\{x_{0}(t): t \geq t_{0}\right\}\right)=0 .
$$

\subsection{Almost automorphic solutions minimizing a subvariant functional}

In the case $p \in(1, \infty)$, we have the following main Theorem.

Theorem 3.2 Let $f \in A A S^{p} U(\mathbb{R} \times X, X)$ and $K$ be a compact subset of $X$. Assume that (H1)(H3) are satisfied. Moreover, if equation (1.1) has at least a mild solution $x_{0}$ defined and bounded on $\left[t_{0},+\infty\right)$. Then, the following hold:

(i) If $\lambda_{K}$ is a subvariant functional associated to the compact set $K$, then equation (1.1) has at least a minimal $K$-valued solution $x$.

(ii) If equation (1.1) has a unique minimal $K$-valued solution $x$, then $x$ is compact almost automorphic. 
Proof. Let $K:=\overline{\left\{x_{0}(t): t \geq t_{0}\right\}}$, by Lemma 3.6 the set $K$ is compact in $X$.

(i) Define $\delta=\inf _{x \in \mathcal{F}_{K}} \lambda_{K}(x)$. Using Lemma 3.5, we claim that the set $\mathcal{F}_{K}$ is nonempty.

Hence, there exists a sequence $\left(x_{n}\right)_{n} \subset \mathcal{F}_{K}$, such that

$$
\lim _{n \rightarrow+\infty} \lambda_{K}\left(x_{n}\right)=\delta .
$$

Given $U_{n}:=\left\{x_{n}: n \in \mathbb{N}\right\} \subset C(\mathbb{R}, X)$, by definition of $\mathcal{F}_{K}$, the set $U_{n}(t):=\left\{x_{n}(t), n \in \mathbb{N}\right\} \subset K$, for each $t \in \mathbb{R}$.

Since $f \in A A S^{p} U(\mathbb{R} \times X, X)$, it follows by Theorem 3.1, that tha family $U_{n}$ is equicontinuous. Hence, in view of Arzela-Ascoli Theorem, we claim that $U_{n}$ is a relatively compact subset of $C(\mathbb{R}, X)$ endowed with the topology of compact convergence. Therefore, there exists a subsequence of $\left(x_{n}\right)_{n}$ (steel denoted by $\left.\left(x_{n}\right)_{n}\right)$ such that

$$
x_{n}(t) \rightarrow x_{*}(t) \quad \text { as } n \rightarrow+\infty,
$$

uniformly on each compact subset of $\mathbb{R}$. Obviously, $x_{*} \in C(\mathbb{R}, X)$ with range including in $K$ (i.e., $\left.x_{*} \in C_{K} \mathbb{R}, X\right)$ ).

Since, for every $n \in \mathbb{N}, x_{n}$ is a mild solution on $\mathbb{R}$ of equation (1.1), we claim (using the same proof as in Lemma 3.5) that

$$
x_{*}(t)=T(t-s) x_{*}(s)+\int_{s}^{t} T(t-\sigma) f\left(\sigma, x_{*}(\sigma)\right) d \sigma \quad \text { for } t \geq s .
$$

Thus $x_{*}$ is a mild solution on $\mathbb{R}$ of equation (1.1). This implies that $x_{*} \in \mathcal{F}_{K}$ and that

$$
\delta \leq \lambda_{K}\left(x_{*}\right) .
$$

By (3.27) and Definition 2.7, we obtain that

$$
\lambda_{K}\left(x_{*}\right) \leq \liminf _{n \rightarrow+\infty} \lambda_{K}\left(x_{n}\right) .
$$

From (3.27), (3.28) and (3.29), we deduce that

$$
\lambda_{K}\left(x_{*}\right)=\delta .
$$

Consequently, $x_{*}$ is a minimal $K$-valued solution:

$$
\lambda_{K}\left(x_{*}\right)=\inf _{x \in \mathcal{F}_{K}} \lambda_{K}(x) .
$$

This proves the existence of the minimal $K$-valued solution of equation (1.1).

(ii) Let $x_{*}$ be the unique minimal solution with relatively compact range of equation (1.1). We show that $x_{*}$ is compact almost automorphic. In fact, let $\left(t_{n}^{\prime}\right)_{n}$ be a sequence of real numbers. Since $f \in A A S^{p} U(\mathbb{R} \times X, X)$ it follows that there exist a subsequence $\left(t_{n}\right)_{n} \subset\left(t_{n}^{\prime}\right)_{n}$ and a function $g: \mathbb{R} \times X \longrightarrow X$ such that (2.3) holds.

Define the set $U_{n}=\left\{x_{n}: n \in \mathbb{N}\right\} \subset C(\mathbb{R}, X)$ where for each $t \in \mathbb{R}, x_{n}(t):=x_{*}\left(t+t_{n}\right)$.

By assumption, for each $t \in \mathbb{R}, x_{n}(t) \in K$. Hence, for each $t \in \mathbb{R}$

$$
U_{n}(t) \subset K .
$$

Moreover, by Theorem 3.1, it holds in view of Arzela-Ascoli Theorem in $C(\mathbb{R}, X)$ endowed with topology of compact convergence that, there exist $y_{*} \in C(\mathbb{R}, X)$ and a subsequence $\left(t_{n}\right)$ such that

$$
x_{n}(t) \rightarrow y_{*}(t) \text { as } n \rightarrow \infty,
$$

uniformly on compact subsets of $\mathbb{R}$. Therefore,

$$
T(t-s) x_{n}(s) \rightarrow T(t-s) y_{*}(s) \text { as } n \rightarrow \infty,
$$

uniformly on compact subsets of $\mathbb{R}$. On the other hand, since $x_{*}$ is a mild solution of equation (1.1), we have

$$
x_{*}\left(t+t_{n}\right)=T(t-s) x_{*}\left(s+t_{n}\right)+\int_{s}^{t} T(t-\sigma) f\left(\sigma+t_{n}, x_{*}\left(\sigma+t_{n}\right)\right) d \sigma, \quad t \geq s .
$$


Then, using the same reasoning as in Lemma 3.5, we claim that

$$
y_{*}(t)=T(t-s) y_{*}(s)+\int_{s}^{t} T(t-\sigma) f\left(\sigma+t_{n}, y_{*}(\sigma)\right) d \sigma, \quad t \geq s .
$$

Consequently, $y_{*}$ is a mild solution on $\mathbb{R}$ with relatively compact range in $K$ of equation

$$
x^{\prime}(t)=A x(t)+g(t, x(t)), \quad t \in \mathbb{R} .
$$

By definition of the subvariant $\lambda_{K}$, we obtain that $\lambda_{K}\left(y_{*}\right) \leq \lambda_{K}\left(x_{*}\right)$. Then, from (3.31) we deduce that

$$
\lambda_{K}\left(y_{*}\right) \leq \inf _{x \in \mathcal{F}_{K}} \lambda_{K}(x)
$$

Furthermore, since $y_{*}$ is a mild solution with relatively compact range in $K$ of equation (3.32), it follows in view of (2.3) and by using the same reasoning as for $x_{*}$ by taking the sequence $\left(-t_{n}\right)_{n}$ instead of $\left(t_{n}\right)_{n}$, that there exists a mild solution $z_{*}$ with relatively compact range in $K$ of equation (1.1). Then, we have

$$
\lambda_{K}\left(z_{*}\right) \leq \lambda_{K}\left(y_{*}\right)
$$

Therefore, by (3.33), we obtain

$$
\lambda_{K}\left(z_{*}\right) \leq \inf _{x \in \mathcal{F}_{K}} \lambda_{K}(x)
$$

Hence

$$
\lambda_{K}\left(z_{*}\right)=\inf _{x \in \mathcal{F}_{K}} \lambda_{K}(x) .
$$

Therefore, $z_{*}$ is a minimal solution with relatively compact range in $K$ of equation (1.1). By uniqueness we deduce that $x_{*}=z_{*}$. Consequently, $x_{*}$ is compact almost automorphic.

From Theorem 3.2, we deduce the following result.

Corollary 3.1 Let $f \in A A S^{p} U(\mathbb{R} \times X, X)$. Assume that (H1)-(H3) are satisfied. If equation (1.1) has a unique bounded mild solution $x$ on $\mathbb{R}$, then $x$ is compact almost automorphic.

Proof. By assumptions, all hypotheses of Theorem 3.2 are satisfied and in particular, the set $K:=\overline{\{x(t): t \geq 0\}}$ is compact. Hence, by taking $\lambda_{K} \equiv 1$, we conclude the result in view of Theorem 3.2 (ii).

For $p=1$, Theorem 3.2 and Corollary 3.1 become :

Theorem 3.3 Let $K$ be a compact subset of $X$. Assume that (H1) (H2) and (H4) are satisfied. Moreover, if equation (1.1) has at least a mild solution $x_{0}$ defined and bounded on $\left[t_{0},+\infty\right)$. Then, the following hold:

(i) If $\lambda_{K}$ is a subvariant functional associated to the compact set $K$, then equation (1.1) has at least a minimal $K$-valued solution $x$.

(ii) If equation(1.1) has a unique minimal $K$-valued solution $x$, then $x$ is compact almost automorphic.

Proof. We argue as the same as in the proof of Theorem 3.2, using Remarks 3.1 , 3.2 and Lemma 3.8 in place of Lemma 3.6 .

Hence, we have the following Corollary.

Corollary 3.2 Let (H1), (H2) and (H4) be satisfied. If equation (1.1) has a unique bounded mild solution $x$, then $x$ is compact almost automorphic.

\section{Application}

Consider the following nonautonomous reaction-diffusion problem :

$$
\left\{\begin{array}{l}
\frac{\partial}{\partial t} v(t, \xi)=\sum_{k=1}^{n} \frac{\partial^{2}}{\partial \xi_{i}^{2}} v(t, \xi)+g(v(t, \xi))+h(t, \xi) \text { for } t \in \mathbb{R} \text { and } \xi \in \Omega \\
v(t, \xi)=0 \text { for } t \in \mathbb{R}, \xi \in \partial \Omega
\end{array}\right.
$$


where $\Omega \subset \mathbb{R}^{n}, n \geq 1$ is bounded and open with smooth boundary $\partial \Omega, g: \mathbb{R} \rightarrow \mathbb{R}$ and $h: \mathbb{R} \times \Omega \rightarrow$ $\mathbb{R}$.

Given the Banach space $X=C_{0}(\bar{\Omega}):=\left\{x \in C(\bar{\Omega}, \mathbb{R}):\left.x\right|_{\partial \Omega}=0\right\}$ endowed with the uniform norm topology. Let

$$
h(t, \xi)=\sin \left(\frac{1}{2+\cos t+\cos \sqrt{2} t}\right) h_{0}(\xi)+a(t) \text { for } t \in \mathbb{R} \text { and } \xi \in \Omega
$$

where $h_{0} \in X$ and $a(t)=\sum_{n \geq 1} \beta_{n}(t)$ such that for every $n \geq 1$

$$
\beta_{n}(t)=\sum_{i \in P_{n}} H\left(n^{2}(t-i)\right),
$$

with $P_{n}=3^{n}(2 \mathbb{Z}+1)$ and $H \in C_{0}^{\infty}(\mathbb{R}, \mathbb{R})$ with support in $\left(\frac{-1}{2}, \frac{1}{2}\right)$ such that

$$
H \geq 0, \quad H(0)=1 \quad \text { and } \quad \int_{\frac{-1}{2}}^{\frac{1}{2}} H(s) d s=1 .
$$

Remark 4.1 25] The function $a \in C^{\infty}(\mathbb{R}, \mathbb{R})$ but $a \notin A A(\mathbb{R}, \mathbb{R})$ since it is not bounded on $\mathbb{R}$. However $a \in A A S^{1}(\mathbb{R}, \mathbb{R})$.

Define the operator $A: D(A) \subset X \rightarrow X$ by

$$
\left\{\begin{array}{l}
D(A)=\left\{x \in X \cap H_{0}^{1}(\Omega): \sum_{k=1}^{n} \frac{\partial^{2}}{\partial \xi_{i}^{2}} x:=\Delta x \in X\right\}, \\
A x=\Delta x .
\end{array}\right.
$$

It is well known that $(A, D(A))$ generates a compact $C_{0}$-semigroup $(T(t))_{t \geq 0}$ on $X$ such that

$$
\|T(t)\| \leq M e^{-\lambda_{1} t} \quad \text { for } t \geq 0,
$$

where $\lambda_{1}:=\min \{\lambda: \lambda \in \sigma(-A)\}>0$, see [17] for more details.

Consequently, hypotheses (H1) and (H2) are satisfied. Moreover, we assume that $g$ is locally Lipschitzian such that

$$
g(0)=0 \text { and } \limsup _{|r| \rightarrow+\infty} \frac{g(r)}{r}<\lambda_{1} .
$$

Remark 4.2 Condition (4.3) implies: there exists $\bar{M} \geq 0$ such that $r g(r) \leq C r^{2}$ for $|r| \geq \bar{M}$ with $C<\lambda_{1}$.

Consider $G: X \rightarrow X$ and $H: \mathbb{R} \rightarrow X$ defined by

$$
G(x)(\xi)=g(x(\xi)), \quad x \in X, \xi \in \Omega
$$

and

$$
H(t)(\xi)=h(t, \xi), \quad x \in X, \xi \in \Omega .
$$

Hence, we denote by $f: \mathbb{R} \times X \rightarrow X$ the function defined by

$$
f(t, x)=G(x)+H(t), \quad t \in \mathbb{R}, x \in X .
$$

Then, equation (4.1) is equivalent to the following abstract evolution equation:

$$
x^{\prime}(t)=A x(t)+f(t, x(t)) \quad \text { for } t \in \mathbb{R} .
$$

The corresponding initial value problem is the following:

$$
\left\{\begin{array}{l}
x^{\prime}(t)=A x(t)+f(t, x(t)) \quad \text { for } t \geq 0 \\
x(0)=x_{0},
\end{array}\right.
$$

Now, under the above assumptions we prove the existence of a unique bounded global solution to equation (4.6) under the weak assumption that $H$ (and hence $f$ ) is not bounded on $t$, see Remark 4.1 
Theorem 4.1 For each $x_{0} \in X$ there exist $t\left(x_{0}\right)>0$ and a unique maximal mild solution $x\left(\cdot, x_{0}\right)$ of equation (4.6) defined on $\left[0, t\left(x_{0}\right)\right)$ such that

$$
t\left(x_{0}\right)=+\infty \quad \text { or } \quad \limsup _{t \rightarrow t\left(x_{0}\right)}\left\|x\left(t, x_{0}\right)\right\|=+\infty .
$$

Proof. Fix $R_{0}>0$ and take $t_{0}:=\log \left(2 M \frac{\left(L_{R_{0}}+\lambda_{1} \tilde{M}+\lambda_{1}\right)}{\lambda_{1}}\right) \lambda_{1}^{-1}>0$ where $\tilde{M}:=e^{\left[t_{0}\right]+1}\left(\left[t_{0}\right]+\right.$ 2) $\|f(\cdot, 0)\|_{B S^{1}}<\infty$.

$$
E=\left\{u \in C\left(\left[0, t_{0}\right], X\right): u(0)=x_{0} \text { and }\|u(t)\| \leq R_{0} \text { for all } t \in\left[0, t_{0}\right]\right\} \subset C\left(\left[0, t_{0}\right], X\right) .
$$

The set $E$ equipped with the uniform norm topology is complete since it is closed in the Banach space $\left(C\left(\left[0, t_{0}\right], X\right),\|\cdot\|_{\infty}\right)$. Define the functional $\Phi: E \longrightarrow C\left(\left[0, t_{0}\right], X\right)$ by

$$
\Phi(u)(t)=T(t) x_{0}+\int_{0}^{t} T(t-s) f(s, u(s)) d s \quad \text { for } t \in\left[0, t_{0}\right] .
$$

Let $u \in E$, we show that $\Phi(u) \in E$. In fact,

$$
\begin{aligned}
\|\Phi(u)(t)\| & \leq M e^{-\lambda_{1} t_{0}}\left\|x_{0}\right\|+M \int_{0}^{t} e^{-\lambda_{1}(t-s)}\|f(s, u(s))\| d s \\
& \leq M e^{-\lambda_{1} t_{0}} R_{0}+M \int_{0}^{t} e^{-\lambda_{1}(t-s)}[\|f(s, u(s))-f(s, 0)\|+\|f(s, 0)\|] d s \\
& \leq M e^{-\lambda_{1} t_{0}} R_{0}+M e^{-\lambda_{1} t_{0}} \int_{0}^{t} e^{\lambda_{1} s} L_{R_{0}}\|u(s)\| d s+M e^{-\lambda_{1} t_{0}} \sum_{k=0}^{\left[t_{0}\right]+1} \int_{k}^{k+1} e^{\lambda_{1} s}\|f(s, 0)\| d s \\
& \leq e^{-\lambda_{1} t_{0}} M\left(1+\frac{L_{R_{0}}}{\lambda_{1}}+\tilde{M}\right) R_{0}=\frac{R_{0}}{2} \leq R_{0} \quad \text { for all } t \in \mathbb{R} .
\end{aligned}
$$

Then $\Phi(E) \subset E$. Now, let $u, v \in E$, then we have

$$
\begin{aligned}
\|\Phi(u)(t)-\Phi(v)(t)\| & \leq M \int_{0}^{t} e^{-\lambda_{1}(t-s)}\|f(s, u(s))-f(s, v(s))\| d s \\
& \leq e^{-\lambda_{1} t_{0}} M \frac{L_{R_{0}}}{\lambda_{1}} \\
& \leq e^{-\lambda_{1} t_{0}} M\left(\frac{L_{R_{0}}}{\lambda_{1}}+\tilde{M}+1\right)\|u-v\|_{\infty}=\frac{\|u-v\|_{\infty}}{2} \quad \text { for all } t \in \mathbb{R} .
\end{aligned}
$$

Hence

$$
\|\Phi(u)-\Phi(v)\|_{\infty} \leq \frac{\|u-v\|_{\infty}}{2}
$$

which proves that $\Phi$ is a contraction map. By Banach fixed point Theorem, we deduce the existence of a unique mild solution $x_{0}$ to equation (4.6) defined on $\left[0, t_{0}\right]$. Let $t_{\varepsilon}>t_{0}$ be well chosen and consider equation (4.6) for $t \in\left[t_{0}, t_{\varepsilon}\right]$ with initial data $x_{t_{0}}=x_{0}\left(t_{0}\right)$. Arguing as above, we can prove the existence of a unique mild solution $x_{\varepsilon}:\left[t_{0}, t_{\varepsilon}\right] \longrightarrow X$ with $x_{\varepsilon}\left(t_{0}\right)=x_{t_{0}}$. Then, we define

$$
x= \begin{cases}x_{0} & \text { in }\left[0, t_{0}\right], \\ x_{\varepsilon} & \text { in }\left[t_{0}, t_{\varepsilon}\right],\end{cases}
$$

By construction $x:\left[0, t_{\varepsilon}\right] \longrightarrow X$ is continuous. Therefore $x$ is the unique solution of equation (4.6) in $\left[0, t_{\varepsilon}\right]$. Hence, we adopt the same extension technique and we prove the existence of a unique maximal solution $x:\left[0, t\left(x_{0}\right)\right) \longrightarrow X$ where $t\left(x_{0}\right):=\sup \left\{t_{0}>0\right.$ : there exist $x \in$ $C\left(\left[0, t_{0}\right], X\right)$ solution to (4.6) $\}$. Now, we show formula (4.1). By contradiction, assume that $t\left(x_{0}\right)<$ $\infty$ and there exists $C \geq 0$ such that $\|x(t)\| \leq C$ for all $t \in\left[0, t\left(x_{0}\right)\right)$. We show that $x$ is uniformly continuous on $\left[0, t\left(x_{0}\right)\right)$. Let $\left(t_{n}\right)_{n},\left(s_{n}\right)_{n} \in\left[0, t\left(x_{0}\right)\right)$ be two sequences such that $\sigma_{n}=t_{n}-s_{n} \rightarrow 0$ as $n \rightarrow \infty$. Without loss of generality we assume that $0 \leq \sigma_{n} \leq 1$ for all $n \geq 0$. Hence, we have

$$
\left\|T\left(t_{n}\right) x_{0}-T\left(s_{n}\right) x_{0}\right\| \leq M\left\|T\left(\sigma_{n}\right) x_{0}-x_{0}\right\| \rightarrow 0 \text { as } n \rightarrow \infty
$$

follows from the strong continuity of the semigroup $(T(t))_{t \geq 0}$ on $X$. Moreover, we have

$$
\begin{aligned}
& \int_{0}^{t_{n}} T\left(t_{n}-s\right) f(s, x(s)) d s-\int_{0}^{s_{n}} T\left(s_{n}-s\right) f(s, x(s)) d s \\
= & \int_{0}^{\sigma_{n}} T\left(t_{n}-s\right) f(s, x(s)) d s-\int_{0}^{s_{n}} T\left(s_{n}-s\right)\left[f(s, x(s))-f\left(s-\sigma_{n}, x\left(s-\sigma_{n}\right)\right] d s\right.
\end{aligned}
$$


Note that by local Lipschitzity of $g$ it holds that $f$ is also locally Lipschitzian on $X$ and it is continuous on $\in\left[0, t\left(x_{0}\right)\right]$ which implies that

$$
\sup _{(t, x) \in\left[0, t\left(x_{0}\right)\right] \times \bar{B}(0, C)}\|f(t, x)\|=\tilde{C}<\infty .
$$

Hence,

$$
\left\|T\left(t_{n}-s\right) f(s, x(s))\right\| \leq M e^{\lambda_{1} t\left(x_{0}\right)} \tilde{C} \quad \text { for all } s \in\left[0, t\left(x_{0}\right)\right)
$$

Then, by dominated convergence Theorem we claim that $\int_{0}^{\sigma_{n}} T\left(t_{n}-s\right) f(s, x(s)) d s \rightarrow 0$ as $n \rightarrow \infty$. Furthermore,

$$
\begin{aligned}
& \left\|\int_{0}^{s_{n}} T\left(s_{n}-s\right)\left[f(s, x(s))-f\left(s-\sigma_{n}, x\left(s-\sigma_{n}\right)\right)\right] d s\right\| \leq \\
& M_{\lambda_{1}} \int_{0}^{t\left(x_{0}\right)}\left[\left\|f(s, x(s))-f\left(s, x\left(s-\sigma_{n}\right)\right)\right\|+\left\|f\left(s, x\left(s-\sigma_{n}\right)\right)-f\left(s-\sigma_{n}, x\left(s-\sigma_{n}\right)\right)\right\|\right] d s \leq \\
& M_{\lambda_{1}} L_{C} \int_{0}^{t\left(x_{0}\right)}\left\|x(s)-x\left(s-\sigma_{n}\right)\right\| d s+M_{\lambda_{1}} \int_{0}^{t\left(x_{0}\right)}\left\|f\left(s, x\left(s-\sigma_{n}\right)\right)-f\left(s-\sigma_{n}, x\left(s-\sigma_{n}\right)\right)\right\| d s
\end{aligned}
$$

where $M_{\lambda_{1}}=M e^{\lambda_{1} t\left(x_{0}\right)}$. By the continuity of $x$, it follows using dominated convergence Theorem that

$$
\begin{gathered}
\int_{0}^{t h a t}\left\|x(s)-x\left(s-\sigma_{n}\right)\right\| d s ; \int_{0}^{t\left(x_{0}\right)}\left\|f\left(s, x\left(s-\sigma_{n}\right)\right)-f\left(s-\sigma_{n}, x\left(s-\sigma_{n}\right)\right)\right\| d s \rightarrow 0 \text { as } n \rightarrow \infty \text {. Thus } \\
\left\|\int_{0}^{t_{n}} T\left(t_{n}-s\right) f(s, x(s)) d s-\int_{0}^{s_{n}} T\left(s_{n}-s\right) f(s, x(s)) d s\right\| \rightarrow 0 \text { as } n \rightarrow \infty
\end{gathered}
$$

Consequently, by (4.8) and (4.9) we claim that

$$
\left\|x\left(t_{n}\right)-x\left(s_{n}\right)\right\| \rightarrow 0 \text { as } n \rightarrow \infty .
$$

Then, $x$ is uniformly continuous on $\left[0, t\left(x_{0}\right)\right)$ which implies that $\lim _{t \rightarrow t\left(x_{0}\right)^{-}} x(t)=x_{t\left(x_{0}\right)} \in X$. Therefore, there exists $\eta>0$ such that $y$ is solution to equation (4.6) in $\left[T\left(x_{0}\right), T\left(x_{0}\right)+\eta\right]$ with initial data $y\left(T\left(x_{0}\right)\right)=x_{t\left(x_{0}\right)}$. Hence

$$
z= \begin{cases}x & \text { in }\left[0, t\left(x_{0}\right)\right] \\ y & \text { in }\left[t\left(x_{0}\right), t\left(x_{0}\right)+\eta\right]\end{cases}
$$

defines a solution of equation (4.6) in $\left[0, t\left(x_{0}\right)+\eta\right]$ which contradicts the fact that $x$ is maximal. Consequently, formula (4.1) is satisfied.

Lemma 4.1 [17, Theorem 8.3.7] Assume $H=0$. Then, for each $x_{0} \in X$ the following hold (i) Equation (4.6) has a unique global solution $u_{x}$ given by:

$$
u_{x}(t)=T(t) x_{0}+\int_{0}^{t} T(t-s) G(x(s)) d s, \quad t \geq 0 .
$$

(ii) The solution $u_{x}$ is bounded on $\mathbb{R}^{+}$i.e., $\sup _{t \geq 0}\left\|u_{x}(t)\right\|<\infty$.

Theorem 4.2 Let $p \in[1, \infty)$. Then for each $x_{0} \in X$ equation (4.6) has a unique global bounded mild solution $x$ given by

$$
x(t)=T(t) x_{0}+\int_{0}^{t} T(t-s) G(x(s)) d s+\int_{0}^{t} T(t-s) H(s) d s, \quad t \geq 0 .
$$

Proof. Fix $x_{0} \in X$ and let $x$ be the unique maximal solution to (4.6) defined on $\left[0, t\left(x_{0}\right)\right)$. Moreover, using Theorem 4.1 $x$ satisfies formula (4.1). Note that a mild solution of equation (4.6) is given by

$$
x(t)=u_{x}(t)+\int_{0}^{t} T(t-s) H(s) d s, \quad t \in\left[0, t\left(x_{0}\right)\right) .
$$


First, we prove that $x$ is global. In fact, by Lemma 4.17(i), it holds that $\sup _{t \in\left[0, t\left(x_{0}\right)\right)}\left\|u_{x}(t)\right\|<\infty$, if it is not, $t\left(x_{0}\right)<\infty$ which contradicts the fact that $u_{x}$ is global. Hence

$$
\begin{aligned}
\|x(t)\| & \leq\left\|u_{x}(t)\right\|+\int_{0}^{t}\|T(t-s) H(s)\| d s \\
& \leq\left\|u_{x}(t)\right\|+M \int_{0}^{t} e^{-\lambda_{1}(t-s)}\|H(s)\| d s \\
& \leq\left\|u_{x}(t)\right\|+M \sum_{k=0}^{[t]+1} \int_{k}^{k+1} e^{-\lambda_{1}(t-s)}\|H(s)\| d s \\
& \leq \sup _{t \in\left[0, t\left(x_{0}\right)\right)}\left\|u_{x}(t)\right\|+\frac{M e^{3 \lambda_{1}}}{e^{\lambda_{1}}-1}\|H\|_{B S^{p}}, \quad t \in\left[0, t\left(x_{0}\right)\right) .
\end{aligned}
$$

Then

$$
\sup _{t \in\left[0, t\left(x_{0}\right)\right)}\|x(t)\|<\infty .
$$

Consequently, by (4.1), we claim that $\left.t\left(x_{0}\right)\right)=\infty$. Thus $x$ is global.

To conclude, we show that $x$ is bounded on $\mathbb{R}^{+}$. Using Lemma 4.1 (ii) we have

$$
\sup _{t \geq 0}\left\|u_{x}(t)\right\|<\infty
$$

Therefore, by (4.10) we can deduce that

$$
\sup _{t \geq 0}\|x(t)\|<\infty
$$

In order to prove the existence of a compact almost automorphic solution for equation (4.1), we need the following results.

Lemma 4.2 The function $f$ satisfies Hypothesis (H4) with $\phi=1$ and $\psi=H$. That is $f \in$ $A A S^{1} U(\mathbb{R} \times X, X)$.

Proof. Let $R \geq 0$ and $B \subset X$ bounded by $R$. By (4.3), we have

$$
\begin{aligned}
\|G(x)\| & \leq L_{R}\|x\|+\|G(0)\| \\
& \leq L_{R} \cdot R \text { for all } x \in B .
\end{aligned}
$$

This proves the fact for $G$. To conclude, it suffices to prove that $H \in A A S^{1}(\mathbb{R}, X)$. In fact, let $\left(t_{n}^{\prime}\right)_{n} \subset \mathbb{R}$ be a sequence. Since $a \in A A S^{1}(\mathbb{R}, \mathbb{R})$ (see [25]) and $b: t \longmapsto \sin \left(\frac{1}{2+\cos t+\cos \sqrt{2} t}\right) \in$ $A A S^{1}(\mathbb{R}, \mathbb{R})$, it follows that there exist $\left(t_{n}\right)_{n} \subset\left(t_{n}^{\prime}\right)_{n}$ and $\tilde{a}, \tilde{b} \in L^{\infty}(\mathbb{R}, \mathbb{R})$ such that, for each $t \in \mathbb{R}$

$$
\int_{t}^{t+1}\left|a\left(s+t_{n}\right)-\tilde{a}(s)\right| d s \rightarrow 0, \int_{t}^{t+1}\left|b\left(s+t_{n}\right)-\tilde{b}(s)\right| d s \rightarrow 0 \text { as } n \rightarrow \infty .
$$

and

$$
\int_{t}^{t+1}\left|\tilde{a}\left(s-t_{n}\right)-a(s)\right| d s \rightarrow 0, \int_{t}^{t+1}\left|\tilde{b}\left(s-t_{n}\right)-b(s)\right| d s \rightarrow 0 \text { as } n \rightarrow \infty .
$$

Define $\tilde{H}(t)(\xi)=\tilde{b}(t) h_{0}(\xi)+\tilde{a}(t)$ for $t \in \mathbb{R}$ and $\xi \in \Omega$. Hence, by 4.11), we claim that for each $t \in \mathbb{R}$

$\int_{t}^{t+1}\left\|H\left(s+t_{n}\right)-\tilde{H}(s)\right\| d s=\left\|h_{0}\right\| \int_{t}^{t+1}\left|a\left(s+t_{n}\right)-\tilde{a}(s)\right| d s+\int_{t}^{t+1}\left|b\left(s+t_{n}\right)-\tilde{b}(s)\right| d s \rightarrow 0$

as $n \rightarrow \infty$. By the same way, we prove that for each $t \in \mathbb{R}$

$$
\int_{t}^{t+1}\left\|\tilde{H}\left(s-t_{n}\right)-H(s)\right\| d s \rightarrow 0 \text { as } n \rightarrow \infty .
$$

By Remark (3.2)-(a), we conclude that $f \in A A S^{1} U(\mathbb{R} \times X, X)$. 
For the uniqueness we need the following additional assumption on $g$ :

$$
\text { The function } r \longmapsto g(r)-r \text { is nonincreasing on } \mathbb{R} \text {. }
$$

Consider the function $E: X \rightarrow \mathbb{R}$ defined by

$$
E(x)=\frac{1}{2} \int_{\Omega}|x(\xi)|^{2} d \xi .
$$

Lemma 4.3 [9] Let $u$ and $v$ be two mild solutions of equation (4.5). Then the following hold.

(i) The function $t \longmapsto E(u(t)-v(t))$ is nonincreasing on $\mathbb{R}$.

(ii) If $t \longmapsto E(u(t)-v(t))$ is constant on $\mathbb{R}$, then there exists $w_{0} \in X$ such that

$$
\begin{gathered}
u(t)-v(t)=w_{0}, \quad t \in \mathbb{R}, \\
G(u(t))-G(v(t))=-\lambda_{1} w_{0}, \quad t \in \mathbb{R} .
\end{gathered}
$$

Moreover, for all $\theta \in[0,1], \theta u+(1-\theta) v$ is also a mild solution of equation (4.5).

Theorem 4.3 Under the above assumptions. The following statements hold for equation (4.5): (i) Equation (4.5) has a at least one mild solution $x_{1}$ which is compact almost automorphic. If $x_{2}$ is a mild solution which is only almost automorphic, then there exists $w_{0} \in X$ such that

$$
x_{2}(t)=x_{1}(t)+w_{0} \quad \text { for } t \in \mathbb{R} .
$$

Consequently $x_{2}$ is compact almost automorphic.

(ii) If the function $r \rightarrow g(r)-r$ is strictly decreasing on $\mathbb{R}$, then the compact almost mild solution is unique

Proof. We use Theorem 3.3 To prove the existence of a compact almost automorphic solution for equation (4.5). By Lemma 4.2 Hypotheses (H1), (H2) and (H4) are satisfied. In addition, from Proposition 4.2 equation (4.6) has at least a mild solution $x_{0}$ defined and bounded on $[0,+\infty)$. Let $K:=\overline{\left\{x_{0}(t): t \geq 0\right\}}$, by Lemma 3.8, $K$ is compact in $X$. Let $c o(K)$ be the convex hull of the compact set $K$. Then $c o(K)$ is a compact convex subset of $X$. It is clear that $K \subset \operatorname{co}(K)$. Define the subvariant functional $\lambda_{c o(K)}: \mathcal{F}_{c o(K)} \longrightarrow[0, \infty)$ by

$$
\lambda_{c o(K)}(x)=\sup _{t \in \mathbb{R}} E(x(t))
$$

associated to the compact set $c o(K)$. We prove that equation (4.5) has at most a minimal $c o(K)$ valued solution. Let $u$ and $v$ be two minimal $c o(K)$-valued solutions of equation (4.5). Define

$$
\inf _{x \in \mathcal{F}_{c o(K)}} \lambda_{c o(K)}(x):=\delta=\sup _{t \in \mathbb{R}} E(u(t))=\sup _{t \in \mathbb{R}} E(v(t))
$$

Case I. Assume that $E(u(t)-v(t)))=c \in \mathbb{R}^{+}$for all $t \in \mathbb{R}$. By the convexity of $c o(K)$ and Lemma 4.3. the set $\mathcal{F}_{c o(K)}$ is convex too. Then $\frac{1}{2} u+\frac{1}{2} v \in \mathcal{F}_{c o(K)}$. Therefore

$$
\delta \leq \sup _{t \in \mathbb{R}} E\left(\frac{1}{2} u(t)+\frac{1}{2} v(t)\right)
$$

On the other hand, by parallelogram inequality, we have

$$
E\left(\frac{1}{2} u(t)+\frac{1}{2} v(t)\right)+E\left(\frac{1}{2} u(t)-\frac{1}{2} v(t)\right)=\frac{1}{2} E(u(t))+\frac{1}{2} E(v(t)),
$$

Then

$$
\sup _{t \in \mathbb{R}} E\left(\frac{1}{2} u(t)+\frac{1}{2} v(t)\right)+\frac{1}{4} c \leq \frac{1}{2} \sup _{t \in \mathbb{R}} E(u(t))+\frac{1}{2} \sup _{t \in \mathbb{R}} E(v(t)) .
$$

Consequently,

$$
\delta+\frac{1}{4} c \leq \frac{1}{2} \delta+\frac{1}{2} \delta
$$


which implies that $c \leq 0$. Hence, by definition of $E$, we obtain $u=v$.

Case II: Let $\left(t_{n}\right)_{n}$ be a sequence of real numbers such that $\lim _{n \rightarrow+\infty} t_{n}=-\infty$. Since by Lemma 4.2, $f \in A A S^{1} U(\mathbb{R} \times X, X)$. Then, by applying Theorem 3.2 two times for $u$ and $v$ respectively, we claim that there exists a subsequence of $\left(t_{n}\right)_{n}$ such that

$$
\begin{aligned}
u\left(t+t_{n}\right) \rightarrow u_{1}(t) & \text { as } & n \rightarrow+\infty, \\
u_{1}\left(t-t_{n}\right) \rightarrow u_{2}(t) & \text { as } & n \rightarrow+\infty, \\
v\left(t+t_{n}\right) \rightarrow v_{1}(t) & \text { as } & n \rightarrow+\infty, \\
v_{1}\left(t-t_{n}\right) \rightarrow v_{2}(t) & \text { as } & n \rightarrow+\infty,
\end{aligned}
$$

uniformly on each compact subset of $\mathbb{R}$, where $u_{2}$ and $v_{2}$ are two minimal co $(K)$-valued solutions of equation (4.5). Using (4.21)-(4.24), we claim that for each $t \in \mathbb{R}$, we have

$$
\lim _{m \rightarrow+\infty} \lim _{n \rightarrow+\infty} E\left(u\left(t+t_{n}-t_{m}\right)-v\left(t+t_{n}-t_{m}\right)\right)=E\left(u_{2}(t)-v_{2}(t)\right) .
$$

Moreover, since by Lemma 4.3 the function $t \rightarrow E(u(t)-v(t))$ is nonincreasing on $\mathbb{R}$ and $\lim _{n \rightarrow+\infty} t_{n}=$ $-\infty$, it follow that for each $t \in \mathbb{R}$

$$
\lim _{n \rightarrow+\infty} E\left(u\left(t+t_{n}\right)-v\left(t+t_{n}\right)\right)=\sup _{\tau \in \mathbb{R}} E(u(\tau)-v(\tau)) .
$$

Therefore,

$$
E\left(u_{2}(t)-v_{2}(t)\right)=\sup _{\tau \in \mathbb{R}} E(u(\tau)-v(\tau)) .
$$

By (4.25) and Case I, we obtain that $u_{2}=v_{2}$. By definition of $E$, we deduce that

$$
\sup _{\tau \in \mathbb{R}} E(u(\tau)-v(\tau))=0,
$$

Consequently $u=v$ and then equation (4.5) has at most one minimal $c o(K)$-valued solution.

(i) Using Theorem 3.3. we claim that equation (4.5) has at least a compact almost automorphic solution. Let $x_{1}$ be a mild solution which is compact almost automorphic. If $x_{2}$ is mild solution which is almost automorphic, then by (16) and Lemma4.3 respectively, the function $t \rightarrow E\left(x_{1}(t)-\right.$ $\left.x_{2}(t)\right)$ is almost automorphic and nonincreasing. Therefore it is constant on $\mathbb{R}$. Consequently, in view of Lemma 4.3-(ii), we obtain (4.17).

(ii) If the function $r \rightarrow g(r)-r$ is strictly decreasing on $\mathbb{R}$, then the uniqueness of the compact almost automorphic solution results directly from (4.15) and (4.16). 


\section{References}

[1] L. Amerio and G. Prouse, Almost-periodic Functions and Functional Equations, Van Nostrand Reinhold, 1971.

[2] G. Arioli, Long term dynamics of a reaction-diffusion system, Journal of Differential Equations, 235, 298-307, (2007).

[3] J. P. Aubin, Applied Functional Analysis, Vol. 47. John Wiley \& Sons, 2011.

[4] M. Baroun, K. Ezzinbi, K. Khalil and L. Maniar, Pseudo almost periodic solutions for some parabolic evolution equations with Stepanov-like pseudo almost periodic forcing terms, Journal of Mathematical Analysis and Applications, 462, (1), 233-262, (2018).

[5] M. Baroun, K. Ezzinbi, K. Khalil and L. Maniar. Almost automorphic solutions for nonautonomous parabolic evolution equations, Semigroup Forum, Springer DOI :10.1007/s00233019-10045-w (2019).

[6] J. Blot, P. Cieutat and K. Ezzinbi, Measure theory and pseudo almost automorphic functions: New developments and applications, Nonlinear Analysis, 75, 2426-2447, (2012).

[7] S. Bochner, A new approach to almost periodicity, Proceedings of the National Academy of Sciences of the United States of America, 48, (12), 2039-2043, (1962).

[8] H. Bohr, Zur theorie der fastperiodischen funktionen, Acta Mathematica, 46, (1-2), 101-214, (1925).

[9] P. Cieutat and K. Ezzinbi, Almost automorphic solutions for some evolution equations through the minimizing for some subvariant functional, applications to heat and wave equations with nonlinearities, Journal of Functional Analysis, 260.9, 2598-2634, (2011).

[10] T. Diagana, G.M. N'Guérékata, Stepanov-like almost automorphic functions and applications to some semilinear equations, Applicable Analysis, 86(6), 723-733, (2007).

[11] B. Es-sebbar and K. Ezzinbi, Stepanov ergodic perturbations for some neutral partial functional differential equations, Math. Meth. Appl. Sci., 39(8), 1945-1963, (2016).

[12] B. Es-Sebbar, Almost automorphic evolution equations with compact almost automorphic solutions, Comptes Rendus Mathematique, 354.11, 1071-1077, (2016).

[13] B. Es-sebbar, K. Ezzinbi, Almost periodicity and almost automorphy for some evolution equations using Favard's theory in uniformly convex Banach spaces, Semigroup Forum, (2016).

[14] J. Favard, Sur les équations différentielles linéaires à coefficients presque-périodiques, Acta Mathematica, 51.1, 31-81, (1928).

[15] A. M. Fink, Almost automorphic and almost periodic solutions which minimize functional, Tôhoku Math. J., 20.2, 323-332, (1968).

[16] J.A. Goldstein, G.M. N'Guerékata, Almost automorphic solutions of semilinear evolution equations, Proc. Amer. Math. Sci. 133 2401-2408, (2005).

[17] T. Cazenave, and A. Haraux, An introduction to semilinear evolution equations, Oxford University Press on Demand, Vol. 13, 1998.

[18] G. Hetzer and W. Shen, Uniform Persistence, Coexistence, and Extinction in Almost Periodic/Nonautonomous Competition Diffusion Systems, SIAM Journal on Mathematical Analysis, (34)1 204-227, (2002).

[19] V. Lakshmikantham, S. Leela, Nonlinear Differential Equations in Abstract Spaces, International Series in Nonlinear Mathematics: Theory, Methods and Applications, 2. Pergamon Press, Oxford-New York, 1981.

[20] J-A. Langa, J-C. Robinson, A. Rodríguez-Bernal, and A. Suárez, Permanence and Asymptotically Stable Complete Trajectories for Nonautonomous Lotka-Volterra Models with Diffusion, SIAM Journal on Mathematical Analysis, (40)6 2179-2216, (2009). 
[21] G.M. N'Guérékata, Almost Automorphic and Almost Periodic Functions in Abstract Spaces, Kluwer Academic Publishers, New-York, 2001.

[22] G. M. N'Guérékata, Existence and uniqueness of almost automorphic mild solutions to some semilinear abstract differential equations, Semigroup Forum, 69(1), (2004).

[23] F. Rothe, Global Solutions of Reaction-diffusion Systems, Vol. 1072, Springer, 2006.

[24] W. Stepanov, Ueber einige verallgemeinerungen der fastperiodischen functionen, Mathematische Annalen, 95, 473-498, (1926).

[25] M. Tarallo, A Stepanov version for Favard theory, Archiv der Mathematik, 90, 53-59, (2008).

[26] C. Zhang, and Wei Lin, Square-mean pseudo almost automorphic process and its application to stochastic evolution equations, Journal of Functional Analysis, 261.1, 69-89, (2011).

[27] Zheng, Zhe-Ming, and Hui-Sheng Ding, On completeness of the space of weighted pseudo almost automorphic functions, Journal of Functional Analysis 268.10, 3211-3218, (2015).

[28] L. Zhenxin, and K. Sun, Almost automorphic solutions for stochastic differential equations driven by Lévy noise, Journal of Functional Analysis, 266.3, 1115-1149, (2014).

Brahim Es-Sebbar, Cadi Ayyad University, Faculty of Sciences and Technology Gueliz, Marrakech, MOROCCO

E-mail address, B. Es-sebbar: essebbar@live.fr

Khalil Ezzinbi, Department of Mathematics, Faculty of Sciences Semlalia, Cadi Ayyad University, MARrakesh B.P. 2390-40000, Morocco.

E-mail address, K. Ezzinbi: ezzinbi@uca.ac.ma

Kamal Khalil, Department of Mathematics, Faculty of Sciences Semlalia, Cadi Ayyad University, MARrakesh B.P. 2390-40000, Morocco.

E-mail address, K. Khalil (Corresponding author): kamal.khalil@ced.uca.ma April 9, 2020

2010 Mathematics subject classification. Primary 46T20-47J35; Secondary 34C27-35K58. 